\title{
Réflexions sur le portrait royal et son fonctionnement dans l'Égypte pharaonique ${ }^{1}$
}

RÉSumé.- L'intérêt que l'art égyptien porte à la représentation humaine individualisée a suscité d'importants débats au sein de la communauté égyptologique à propos de l'existence même du portrait dans la civilisation pharaonique. Le sujet a souvent - et, en réalité, presque toujours - été envisagé en termes d'opposition entre portrait et image idéale ou image type, s'intégrant dans la problématique plus générale du réalisme et de la relation formelle à la réalité dans l'art de l'Égypte antique. Après une brève analyse du problème sur un plan théorique, l'article propose d'examiner dans le contexte de la pensée égyptienne les concepts qui définissent la notion de portrait. Quelques exemples significatifs sélectionnés dans la statuaire royale sont ensuite étudiés afin de préciser les ressorts et les modalités de l'interaction entre les tendances au portrait et à l'image idéal ou idéelle dans les représentations individualisées de l'Égypte ancienne.

Aвstract.- Ancient Egyptian art's concern with individualized human representation has generated much debate among Egyptologists about the very existence of portraiture in pharaonic society. The issue has often - if not always - been thought of in terms of opposition between portrait and ideal image, being a major topic in the broader question of realism and formal relation to reality in Ancient Egyptian art. After a brief analysis of the problem from a theoretical point of view, the article considers the concepts involved in the notion of portrait within the context of Ancient Egyptian thought. Then, a few significant cases selected in royal statuary are investigated in order to elucidate the motives and modalities of the interaction between portrait and ideale image in Ancient Egyptian individualized representations.

\section{PRÉAMBULE: L'ÉGYPTOLOGIE ET LE PROBLÈME DU PORTRAIT DANS L'ART ÉGYPTIEN, OU LES VESTIGES DE L'HYPOTHÈSE - INCONSCIENTE - DU RÉALISME}

Depuis la Renaissance, le regard occidental sur l'art, en général, et le portrait plastique, en particulier, est formaté par le modèle de l'art gréco-romain comme référence suprême du réalisme. C'est ainsi qu'en 1764, Johann Joachim Winckelmann, généralement considéré comme l'un des pionniers de l'historiographie moderne de l'art antique, jugeait avec condescendance l'art égyptien, écrivant:

Les Égyptiens sont restés assez proches de leur style le plus ancien, et chez eux, l'art n'a pu atteindre les sommets auxquels il est parvenu chez les Grecs. (...)

(1) Le présent article constitue une version remaniée de ma communication au colloque du GIRI sur le portrait dans les civilisations de l'Antiquité, à Strasbourg, les 7 et 8 mars 2008, et d'une contribution intitulée "Portrait versus ideal image», à paraître dans Willeke WENDRICH (éd.), UCLA Encyclopedia of Egyptology (peer reviewed online publication), Los Angeles, encyclopédie consultable sur l'internet à l'adresse URL http://repositories.cdlib.org/nelc/uee. 
La première cause des particularités de l'art chez les Égyptiens est leur aspect physique qui n'avait pas assez d'attrait pour stimuler les artistes et leur donner un idéal de beauté. Car la nature les avait moins avantagés que les Étrusques et les Grecs, comme le montre une sorte de conformation chinoise des traits physiques que l'on retrouve sur leurs statues mais aussi sur leurs obélisques et leurs pierres gravées, si bien que leurs artistes ne purent rechercher la variété. La même forme se retrouve dans les têtes des personnages peints sur les momies, qui sont, comme chez les Éthiopiens, d'une grande ressemblance avec le mort, car dans la préparation des corps morts, les Égyptiens cherchaient à conserver tout ce qui pouvait servir à les identifier, y compris les cils des paupières ${ }^{2}$

On pourrait penser qu'après les développements de l'art occidental au $\mathrm{XX}^{\mathrm{e}}$ siècle, une telle appréciation, avec tous les présupposés par rapport au nécessaire réalisme qu'elle implique, ne relève plus que de l'histoire de la discipline ${ }^{3}$, mais, pourtant, il faut bien constater qu'en égyptologie, - comme dans bien d'autres disciplines, d'ailleurs, - cette hypothèse du réalisme - souvent inconsciente et donc d'autant plus pernicieuse - sévit encore jusqu'en ce début du XXIe siècle ${ }^{4}$.

Cette lecture «réaliste» de l'art égyptien, qui pollue véritablement la question du portrait en histoire de l'art pharaonique, se trouve confortée, voire démontrée aux yeux de certains, par la volonté d'individualisation manifestement si présente dans la culture de l'Égypte antique ${ }^{5}$, ainsi que par l'argument jugé souvent imparable: «mais, on peut les reconnaître; c'est donc qu'il s'agit bien d'un portrait »! La préservation des momies égyptiennes, qui avait déjà frappé l'imagination de J. J. Winckelmann et offre à l'Égyptologie le privilège, exceptionnel au sein des sciences historiques, de pouvoir examiner "en chair et en os" nombre des grandes figures dont elle traite, contribue encore à la puissance de ce postulat du réalisme, d'autant que, dans quelques cas privilégiés, une certaine ressemblance a pu être mise en évidence entre la dépouille momifiée d'un pharaon particulier et son iconographie ${ }^{6}$.

(2) Cf. J. J. Winckelmann, Histoire de l'art dans l'Antiquité, traduction de Dominique Tassel, Paris, 2005, p. 100-101.

(3) Pour des résumés, avec bibliographie, de l'évolution de la question du portrait dans l'Égypte ancienne au sein de la discipline égyptologique, cf. Cl. Vandersleyen, «Porträt», dans W. Helck et E. Отto (éd.), Lexikon der Ägyptologie 4, Wiesbaden, 1982, col. 1074-1080; et D. Spanel, Through Ancient Eyes: Egyptian Portraiture. An Exhibition Organized for the Birmingham Museum of Art, Birmingham, Alabama, 1988. Les conséquences des développements de l'art dit contemporain sur la compréhension de l'art égyptien avaient été comprises dès le début du XX $\mathrm{X}^{\mathrm{e}}$ siècle, comme l'illustrent les travaux fondamentaux de H. Schäfer, et en particulier son Von ägyptischer Kunst, Leipzig, 1919 (éd. anglaise par J. Baines, sous le titre Principles of Egyptian Art, Oxford, 1974), et son livre, au titre très significatif, Ägyptische und heutige Kunst, Berlin, 1928.

(4) Cf., à ce propos, les plaidoyers de Roland Tefnin contre l'illusion de ce qu'il appelait «l'immédiateté du figuré », notamment dans Tefnin, «Image et histoire. Réflexions sur l'usage documentaire de l'image égyptienne», Chronique d'Égypte 54 (1979), p. 218-244; ID., «Image, écriture, récit. A propos des représentations égyptiennes de la bataille de Qadesh", Annales d'Histoire de l'Art et Archéologie 2 (1980), p. 7-24; ID., "Discours et iconicité dans l'art égyptien ", Annales d'Histoire de l'Art et Archéologie 5 (1983), p. 5-17 (article réédité dans Göttinger Miszellen. Beiträge zur ägyptologischen Diskussion 79 [1984], p. 55-72); et ID., "Éléments pour une sémiologie de l'image égyptienne», Chronique d'Égypte 66 (1991), p. 60-88.

(5) À ce sujet, on se reportera à l'étude magistrale de J. Assmann, «Preservation and Presentation of Self in Ancient Egyptian Portraiture», dans P. Der Manuelian et R.E. Freed (éd.), Studies in Honor of William Kelly Simpson I, Boston, 1996, p. 55-81; et aux explications développées infra.

(6) Cf. Spanel, op. cit., p. 2-3. En réalité, il s’agit essentiellement des deux cas illustrés par cet auteur, à savoir celui de Thoutmosis III (discuté dans la suite du présent article) et celui de Séthi I ${ }^{\mathrm{er}}$ (plus complexe qu'il n'y paraît, lui aussi; cf. infra, n. 63). En outre, les divers commentaires sur ces ressemblances physionomiques entre momies et sculptures sont parfois très contrastés, révélant avant tout la subjectivité et les attentes de l'observateur, en fonction de ses idées préconçues vis-à-vis du concept de portrait; à cet égard, on comparera l'avis de D. Spanel (loc. cit.) et celui de G. Maspero, qui écrivait à propos du visage momifié de Thoutmosis III : «l'aspect en désappointe les admirateurs du conquérant. Les statues qui nous restent de lui, sans le représenter comme un type de beauté mâle, lui prêtaient des traits fins et spirituels: la comparaison avec le cadavre montre qu'elles ont idéalisé le modèle» (Histoire ancienne des peuples de l'Orient classique II, Paris, 1897, p. 289). 
À l'inverse, d'aucuns ont invoqué les tendances fondamentalement sémiotique et idéalisante, ou générique, de l'art égyptien ${ }^{7}$ pour justifier un scepticisme parfois radical vis-à-vis de l'idée même de l'existence du portrait dans l'Égypte antique, attirant l'attention sur les aspects formulaires des représentations individuelles dans cette civilisation. Malgré quelques avis nuancés ${ }^{8}$, ces positions contrastées ont souvent conduit à des conclusions parfaitement contradictoires. Ainsi, pour ne mentionner qu'un seul cas, particulièrement clair (et sur lequel je reviendrai plus loin), l'art du règne du pharaon monothéiste Akhénaton et son chef-d'œuvre le plus célèbre, le buste de Néfertiti, connu sous l'appellation de buste de Berlin (Ägyptisches Museum 21.300), est-il généralement considéré comme ce que l'art égyptien a produit de plus réaliste («the most lifelike of Egyptian art», pour citer le titre d'un article de Rolf Krauss qui remet très sérieusement en question cette conviction) ${ }^{9}$, alors que Jan Assmann, dans son étude, fondamentale pour notre propos, consacrée au thème de la préservation et de la présentation du soi dans le portrait égyptien, s'en sert précisément comme exemple pour définir ce qui, selon lui, doit être qualifié d'idéalisation ${ }^{10}$.

Une telle confusion, au-delà de sa dimension historique, résulte assurément d'un manque flagrant de définition ou, à tout le moins, d'approche théorique des concepts mis en ouvre, et en particulier de ceux, si souvent utilisés, mais trop rarement clarifiés, de portrait, de réalisme et d'idéalisation.

\section{LE CONCEPT DE PORTRAIT ET SES IMPLICATIONS THÉORIQUES}

Le concept de portrait renvoie, dans toutes ses acceptions reçues, à la représentation, dans quelque médium que ce soit, d'un individu spécifique, c'est-à-dire à la représentation individualisée d'un être identifiable (au moins en théorie et par le[s] producteur[s] dudit portrait). Dans le domaine des arts plastiques, il est généralement conçu en opposition à celui d'image idéale ou d'image type ou idéelle ${ }^{11}$, désignant une individualisation figurative et se rapportant à la notion de réalisme en tant que transposition fidèle et exacte de la réalité objective, par contraste avec la notion d'idéalisation. Si cette opposition fondamentale entre réalisme et idéalisation est traditionnellement acceptée et utilisée comme un concept clé en Histoire de l'art, en général et toutes disciplines et sous-disciplines confondues, elle se révèle néanmoins extrêmement problématique d'un point de vue théorique.

(7) Sur ces aspects de l'art pharaonique, cf. le toujours incontournable H. SchÄFER, Von ägyptischer Kunst (op. cit. supra, n. 3); et D. Laboury, "Fonction et signification de l'image égyptienne», Bulletin de la Classe des Beaux-Arts de l’Académie Royale de Belgique 6e série, IX (1998), p. 131-148.

(8) Parmi lesquels on citera Spanel, op. cit.; Fr. Junge, "Versuch zu einer Ästhetik der ägyptischen Kunst", dans M. Eaton-Krauss et E. Graefe (éd.), Studien zur ägyptischen Kunstgeschichte (Hildesheimer Ägyptologische Beiträge 29), Hildesheim, 1990, , p. 4-6; R. Tefnin, Art et magie au temps des pyramides. L'énigme des têtes de "remplacement" (Monumenta Egyptiaca 5), Bruxelles, 1991, p. 69-73; JungE, "Hem-iunu, Anch-ha-ef und die sog. "Ersatzköpfe" ", dans Kunst des Alten Reiches. Symposium im Deutschen Archäologischen Institut Kairo am 29. und 30. Oktober 1991 (Sonderschrift des Deutschen Archäologischen Instituts, Abteilung Kairo 28), Mayence, 1995, p. 103-9; AssmanN, op. cit.; D. LABoury, La statuaire de Thoutmosis III. Essai d'interprétation d'un portrait royal dans son contexte historique (Ægyptiaca Leodiensia 5), Liège, 1998, p. 647-655; S.-A. Ashton et D. Spanel, "Portraiture", dans D.B. Redford (éd.), The Oxford Encyclopedia of Ancient Egypt 3, Le Caire, 2001, p. 55-9

(9) Cf. R. Krauss, "Nefertiti - A Drawing-board of Beauty? The "most lifelike of Egyptian art" is Simply the Embodiment of Numerical Order», Amarna Letters 1 (1991), p. 46-49.

(10) Cf. Assmann, op. cit., p. 68-71.

(11) Image idéale et image type ou idéelle ne doivent pas être confondues, puisque la première se réfère à un idéal, et la seconde à une idée ou un concept; sur cette distinction, cf. les pertinentes remarques d'ID., op. cit., p. 65-71. Cependant, opposées à la notion de portrait, elles ont en commun leur émancipation par rapport à la réalité perceptuelle - ou réalité telle qu'elle est perçue - dans le sens d'une généralisation, d'un rendu plus générique et moins individualisé. 
Tout d'abord, les deux notions sont ancrées dans des concepts philosophiques qui sont loin de faire l'objet d'un consensus, dans la mesure où elles se réfèrent toutes deux à l'idée même de réalité, une question d'ordre métaphysique, qui a suscité moult débats dans l'histoire de l'humanité et reste encore (et probablement pour longtemps) sans réponse définitive, ou en tout cas appropriée aux diverses cultures humaines attestées à ce jour. Et, de ce point de vue, il est évident que la conception que les anciens Égyptiens avaient développée de la réalité était différente de celles générées par notre Occident moderne.

Par ailleurs, sur un plan strictement formel, les conditions précises de l'opposition et la ligne de démarcation entre les deux concepts posent de sérieux problèmes de définition. Ainsi, par exemple, et en se limitant au domaine de l'art pharaonique, il n'est pas rare que des figures anonymes et subsidiaires ou en groupe soient caractérisées par des effets de réel ${ }^{12}$, c'est-à-dire une individualisation apparente, apte à suggérer l'impression de portrait (ou, plus exactement, l'illusion de proximité et de singularité humaines) ${ }^{13}$, mais qui dérive en réalité d'une convention bien connue et très largement attestée dans l'imagerie pharaonique de dissimilation, graphique ou chromatique ${ }^{14}$ (pl. 1). Faut-il considérer de tels cas comme des portraits de catégories sociales ou des portraits types, soit des notions qui tendraient à estomper, voire à effacer, la distinction et l'opposition entre les deux concepts théoriques de portrait et d'image type ou idéale? Inversement, dans l'art égyptien, toujours, toute représentation d'un individu, nommé ou non, est caractérisée - et souvent identifiable et datable - par le style de son époque, et par la conception générique et idéale de l'être humain qui avait cours lors de la réalisation de l'œuvre ${ }^{15}$. Dans ce sens, l'art pharaonique constitue sans doute l'une des plus belles illustrations des faits que, d'une part, l'imitation plastique de la réalité est toujours, inévitablement, sélective et que, d'autre part, tout portrait, de par son caractère artificiel, est au moins contaminé par une image idéale ou idéelle. Il me semble donc préférable d'envisager les deux concepts théoriques de portrait et d'image idéale ou idéelle non pas dans une opposition dichotomique, mais plutôt comme une combinaison vectorielle, c'est-à-dire comme la conjonction de deux vecteurs divergents, au sein de laquelle les deux dimensions en question sont toujours présentes, en proportions variables.

Enfin, si l'opposition conceptuelle - toute cartésienne - entre portrait et image idéale ou image type, entre réalisme et idéalisation, est devenue, historiquement, une notion établie et (plus ou moins) utile dans l'histoire moderne de l'art occidental, il convient certainement de s'interroger sur sa pertinence dans le contexte de la culture pharaonique, dans une civilisation qui a sans cesse et de façon particulièrement cohérente cherché à établir un lien entre réalité et idéalité ${ }^{16}$.

(12) Sur cette notion, fondamentale dans la problématique du portrait et qui fut introduite dans les discussions à l'occasion du colloque du GIRI par Mlle S. Boehringer, cf. R. BARThEs, "L'effet de réel», Communications 11 (1968) (numéro spécial «Recherches sémiologiques: le vraisemblable»), p. 84-89. Je remercie V. Angenot d'avoir attiré mon attention sur cette référence bibliographique.

(13) Sur la relation non nécessaire, et donc la distinction qu'il convient d'opérer, entre individualisation formelle (ou effet de réel) et portrait, dans le contexte particulier de l'art égyptien, cf. les brillantes de remarques de R. Tefnin, op. cit., p. 9-73.

(14) Sur cette convention, cf. H. G. Fischer, L'écriture et l'art de l'Égypte ancienne. Quatre leçons sur la paléographie et l'épigraphie pharaoniques (Essais et conférences du collège de France), Paris, 1986, p. 30-34.

(15) Cf., notamment, Junge, dans Kunst des Alten Reiches. (op. cit. supra, n. 8); et Assmann, op. cit.

(16) En dehors du secteur de l'image et des arts plastiques, on peut illustrer cette propension fondamentale de la pensée égyptienne par l'historiographie pharaonique: si la démarche même de relater des faits historiques suppose, en soi, une emphase particulière sur la singularité de l'événement à évoquer, dans l'Égypte antique, la représentation de ce qui s'est réellement passé est toujours inévitablement structurée par l'idéal idéologique. 


\section{LE POINT DE VUE DES ANCIENS ÉGYPTIENS}

Toute la culture monumentale de l'Égypte pharaonique ${ }^{17}$ manifeste un profond désir de préservation de l'identité personnelle, - en particulier dans une perspective funéraire, - et, de ce fait, une forte conscience du soi individuel. Et, dans ce sens, comme Jan Assmann l'a parfaitement souligné, «Portraiture is by far the most important and productive genre of Egyptian art, just as biography is the most ancient and productive genre of Egyptian literature ${ }^{18}$. Cependant, même en présence de ce principe fondamental d'auto-thématisation, - comme J. Assmann suggère de le caractériser (Selbstthematizierung - self-thematization) ${ }^{19}$, - l'usage de la notion de portrait dans le contexte pharaonique doit être validé par l'évaluation dans le cadre de la pensée et de l'art de l'Égypte antique des deux concepts qui définissent cette notion sur un plan théorique, soit l'identité individuelle, d'une part, et la reconnaissabilité ou le caractère identifiable, d'autre part.

Comme dans bon nombre de civilisations, le mot qui désigne l'image en ancien égyptien, $t w t$, implique la notion de ressemblance, puisqu'il s'apparente étymologiquement à une racine verbale qui signifie «ressembler à, être comme ou en accord (avec) ${ }^{20}$. L'image est donc clairement conçue comme une transposition figurative et ressemblante de son modèle. Toutefois, les nombreuses usurpations de statues seulement réalisées par la re-gravure du nom, sans la moindre adaptation physionomique, la variabilité des effigies d'un même personnage (royal ou privé) ${ }^{21}$ et les généalogies de portraits, dans lesquelles un individu cherche à s'associer iconographiquement et physionomiquement à un prédécesseur (ou un supérieur) ${ }^{22}$, démontrent que le concept de ressemblance iconique était moins contraignant dans la pensée égyptienne que dans les cultures occidentales modernes. J. Assmann suggère de le définir comme un principe de non-confondabilité (Unverwechselbarkeit), c'est-à-dire un caractère identifiable qui pouvait être garanti sur plusieurs plans, ou par la simple présence du nom du personnage portraituré. En outre, on ne peut sousestimer la dimension métaphysique du concept d'image ressemblante: à quoi est-elle censée ressembler? À l'apparence physique et externe - ou phénoménologique - de son modèle, ou à sa véritable réalité, qui peut, selon les cultures et les conceptions philosophiques, se trouver au-delà des apparences directement perceptibles ? $^{23}$

(17) Sur cette notion de culture monumentale, cf. Assmann, «Égypte ancienne - la mémoire monumentale», dans $\mathrm{Ph}$. Gignoux (éd.), La commémoration. Colloque du centenaire de la section des sciences religieuses de l'EPHE (Bibliothèque de l'EPHE, section des sciences religieuses 91), Louvain - Paris, 1988, p. 47-56; ID. et T. HöLSCHER, Kultur und Gedächnis, Francfort, 1988; AssmanN, Stein und Zeit. Mensch und Gesellschaft im alten Ägypten, Munich, 1991, p. 16-58.

(18) Cf. ID., dans Studies Simpson (op. cit. supra, n. 5), p. 55.

(19) Cf. ibidem; et ID., «Sepulkrale Selbstthematisierung im alten Ägypten», dans A. HAHN et V. KAPP (éd.), Selbstthematisierung und Selbstzeugnis: Bekenntnis und Geständnis, Francfort, 1987, p. 196-222.

(20) Cf., notamment, M. Eaton-Krauss, The Representations of Statuary in Private Tombs of the Old Kingdom (Ägyptologische Abhandlungen 39), Wiesbaden, 1984, \$93 (opinion nuancée); Assmann, Stein und Zeit (op. cit. supra, n. 17), p. 141; R. SCHulZ, Die Entwicklung und Bedeutung des kuboiden Statuentypus. Eine Untersuchung zu den sogenannten “Würfelhockern” (Hildesheimer Ägyptologische Beiträge 34), Hildesheim, 1992, p. 701.

(21) Pour quelques exemples, cf. infra.

(22) Cf. la statuaire privée, qui imite généralement les traits prêtés au roi, ou, dans le domaine de la sculpture royale, les exemples évoqués infra.

(23) Sans mentionner ici l'étroite relation, - et, de ce fait, peut-être une certaine forme de perméabilité, - que la pensée égyptienne établissait entre ces deux notions théoriques - et si occidentales - d'apparence extérieure et de réalité intérieure, comme le suggèrent, par exemple, la complémentarité habituelle dans les textes pharaoniques entre $k d$, «la forme extérieure», et $h n w$, «l'intérieur», ou les expressions qui évoquent une qualité psychologique ou un trait de caractère par la description du visage, comme $n f r-h r$, "parfait, beau de visage», pour "clément» ou "bienveillant», śpd-hrr, "pointu de visage», pour «attentif», mais aussi «capable, efficace», etc. 
Quant à la notion pharaonique d'identité individuelle, tout comme l'égyptianité était, à l'évidence, moins une affaire d'origine ethnique que de comportement à l'égyptienne ${ }^{24}$, elle apparaît fondamentalement conçue comme une intégration personnelle, sur un plan comportemental et fonctionnel, dans l'ordre sociétal. En témoignent pleinement l'importance et la persistance des clichés comportementaux qui structurent la quasi-totalité des textes biographiques que nous a livrés l'Égypte ancienne. Ainsi, en d'autres termes, l'individualité d'une personne, avec son nom, sa généalogie et son destin spécifiques, est-elle toujours définie au sein de la structure sociale de la civilisation pharaonique, c'est-à-dire suivant des types et des idéaux sociaux, qui façonnent et souvent estompent ou absorbent l'éventuelle expression d'une unicité ou d'une singularité.

Dans un tel contexte culturel, la traditionnelle opposition entre portrait et image type ou idéale doit être, plus que jamais, conçue et utilisée sur le mode d'une combinaison vectorielle,- comme suggéré ci-dessous, - ou d'une tension dialectique, qui structure et génère différentes formes d'autothématisation, dans le domaine des arts plastiques, comme dans celui de la littérature.

\section{QUELQUES CAS SIGNIFICATIFS}

Les ressorts et modalités de cette interaction entre les tendances au portrait et à l'image idéale ou idéelle dans l'auto-thématisation iconographique en Égypte antique peuvent être illustrés et investigués plus avant par l'analyse des quelques cas significatifs ${ }^{25}$. Ceux qui suivent sont tous empruntés au domaine de la statuaire royale, pour plusieurs raisons convergentes: comme Sally-Ann Ashton et Donald Spanel l'ont fait remarquer, le portrait dans l'art pharaonique «was limited almost exclusively to sculpture ${ }^{26}$; sauf à échelle très réduite, les représentations en trois dimensions permettent un rendu plus détaillé et plus subtil, qui explique probablement pourquoi, en Égypte, elles exercent toujours une influence sur les arts bidimensionnels, et non l'inverse ${ }^{27}$; en quantité, comme en qualité, l'iconographie royale est nettement mieux préservée et attestée que le portrait privé, sans compter qu'elle détermina souvent l'évolution de ce dernier; et, enfin, en tant que représentation d'un individu et, en même temps, d'une institution, - de loin la plus essentielle de la civilisation égyptienne, - les effigies de pharaon soulèvent des problèmes plus complexes et plus intéressants pour notre propos ${ }^{28}$.

\section{Mykérinos - Menkaourê}

Les portraits du roi Mykérinos, - Menkaourê en ancien égyptien, - qui régna sur l'Égypte durant la première moitié du $\mathrm{XXV}^{\mathrm{e}}$ siècle avant notre ère, apparaissent très cohérents, dans la mesure où, d'une part, sa physionomie particulière est aisément reconnaissable d'une statue à

(24) Cf. A. Loprieno, Topos und Mimesis. Zum Ausländer in der Ägyptischen Literatur (Ägyptologische Abhandlungen 48) Wiesbaden, 1988; B. J. Kemp, Ancient Egypt. Anatomy of a Civilization, 2e edition, Londres / New York, 2006, p. 19-59.

(25) Ces cas ont été sélectionnés dans le but de mettre en lumière les caractéristiques propres du portrait - ou autothématisation iconographique - et de son fonctionnement dans la civilisation et la mentalité pharaoniques de façon globale, soit, d'une certaine manière, indépendamment du facteur évolutif. Pour une analyse diachronique de l'art du portrait en l'Égypte antique, révélant des tendances successives vers un réalisme somatique (visant d'abord à la préservation du corps et de son apparence), un réalisme sémiotique (dont le but est de faire passer, par la reproduction des traits, un message concernant la nature du personnage portraituré), une idéalisation (formatant la représentation individuelle à l'aune d'un modèle idéal), etc, le lecteur pourra se reporter, - une fois de plus, - à l'article magistral de J. Assmann, dans Studies Simpson (op. cit. supra, n. 5).

(26) Cf. Ashton et Spanel, op. cit., p. 55.

(27) Cf. Laboury, La statuaire de Thoutmosis III (op. cit. supra, n. 8), p. 633.

(28) Sur cette question, d'un point de vue général et théorique, cf. ID., op. cit., p. 74-77. 

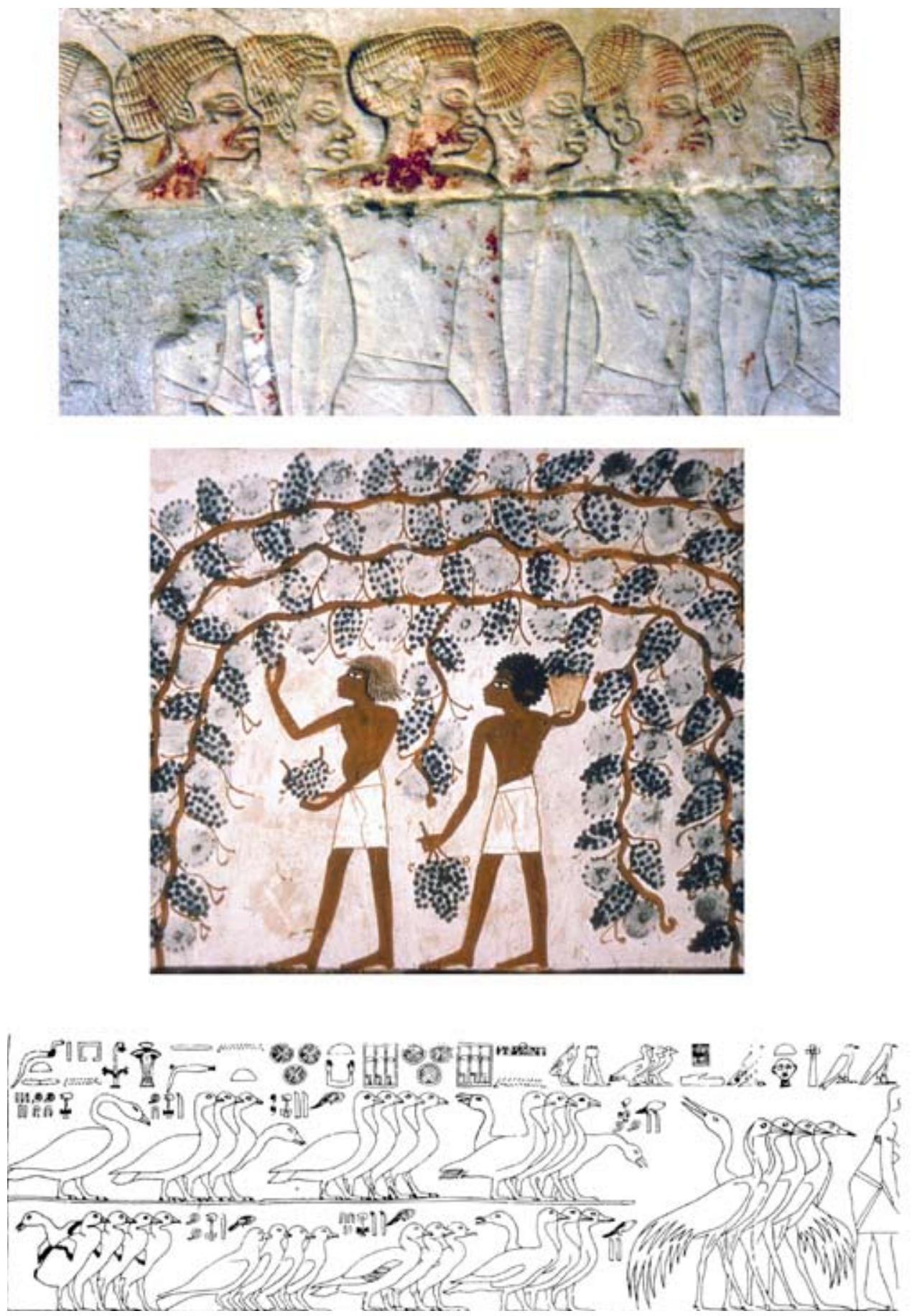

Pl. 1: Effets de réel à des fins de dissimilation dans la tombe de Horemheb à Saqqarah et dans la tombe de Nakht à Cheikh 'Abd el-Gourna (TT 52) (clichés de l'auteur), et illustration du principe de dissimilation appliqué à des figures non humaines (d'après H. G. Fischer, L'écriture et l'art de l'Égypte ancienne. Quatre leçons sur la paléographie et l'épigraphie pharaoniques [Essais et conférences du collège de France], Paris, 1986, p. 32, fig. 4). 


\section{DIMITRI LABOURY}
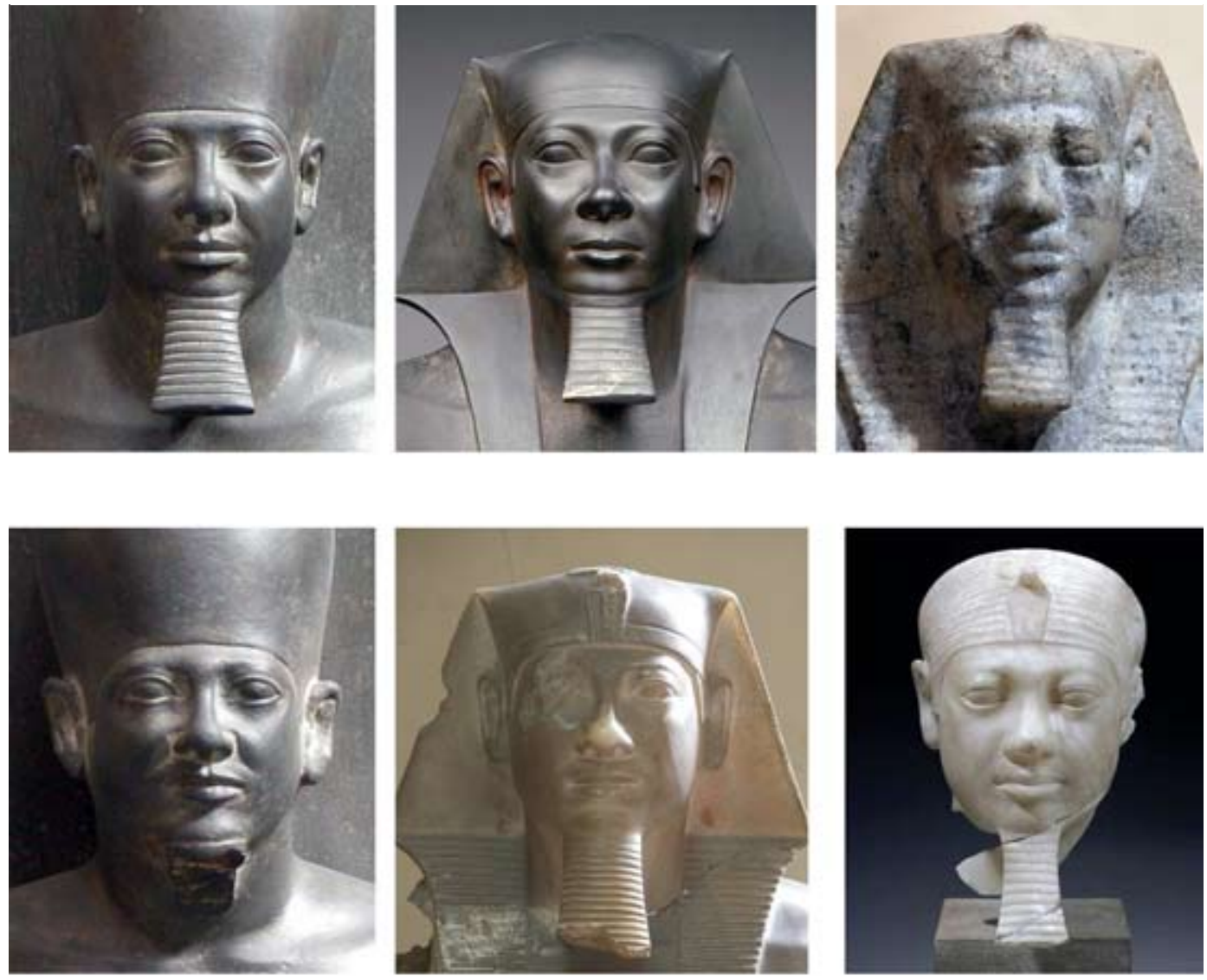

(
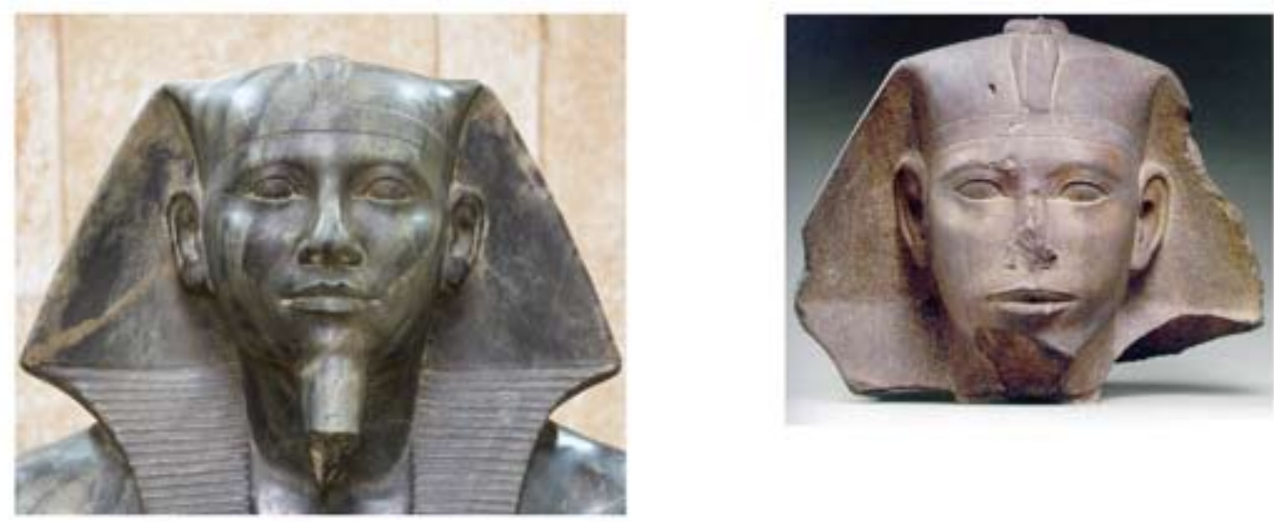

Pl. 2: Comparaison de plusieurs portraits de Mykérinos (Le Caire JE 46499; Boston MFA 11.1738; Le Caire CG 42; JE 40678; JE 40703; Boston MFA 09.203), avec celui de son père, Khéphren (Le Caire CG 14) et celui de son oncle, Radjédef (Louvre E 12626) (clichés de l'auteur et du site internet du MFA Boston et du Louvre). 
LE PORTRAIT ROYAL ET SON FONCTIONNEMENT DANS L'ÉGYPTE PHARAONIQUE
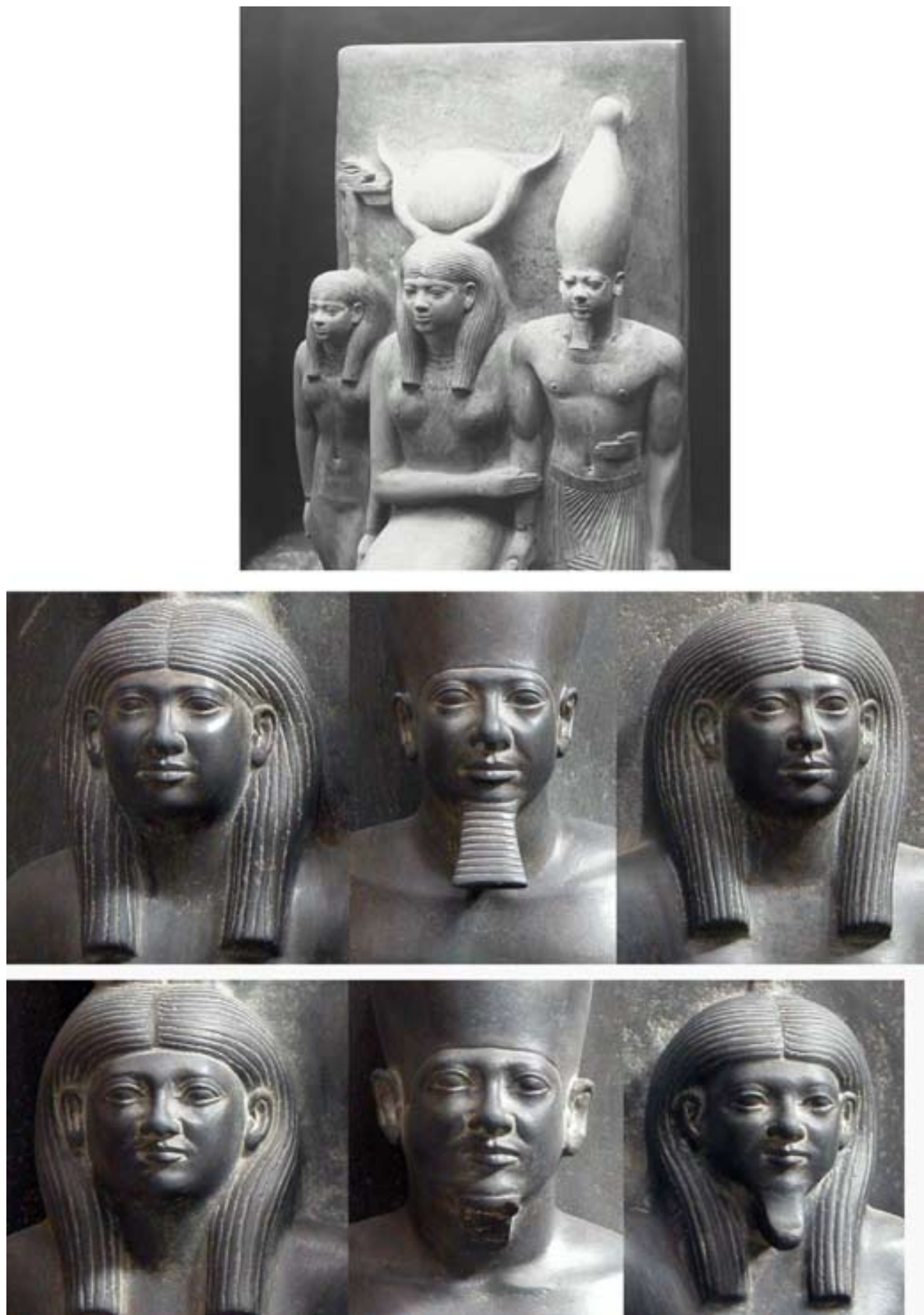

Pl. 3 : la triade de Mykérinos Boston MFA 09.200 (d'après G. A. ReIsner, Mycerinus, Cambridge, MA, 1931 pl. 39) et comparaison des trois visages des triades de Mykérinos Le Caire JE 46499 et JE 40678 (clichés de l'auteur). 


\section{DIMITRI LABOURY}
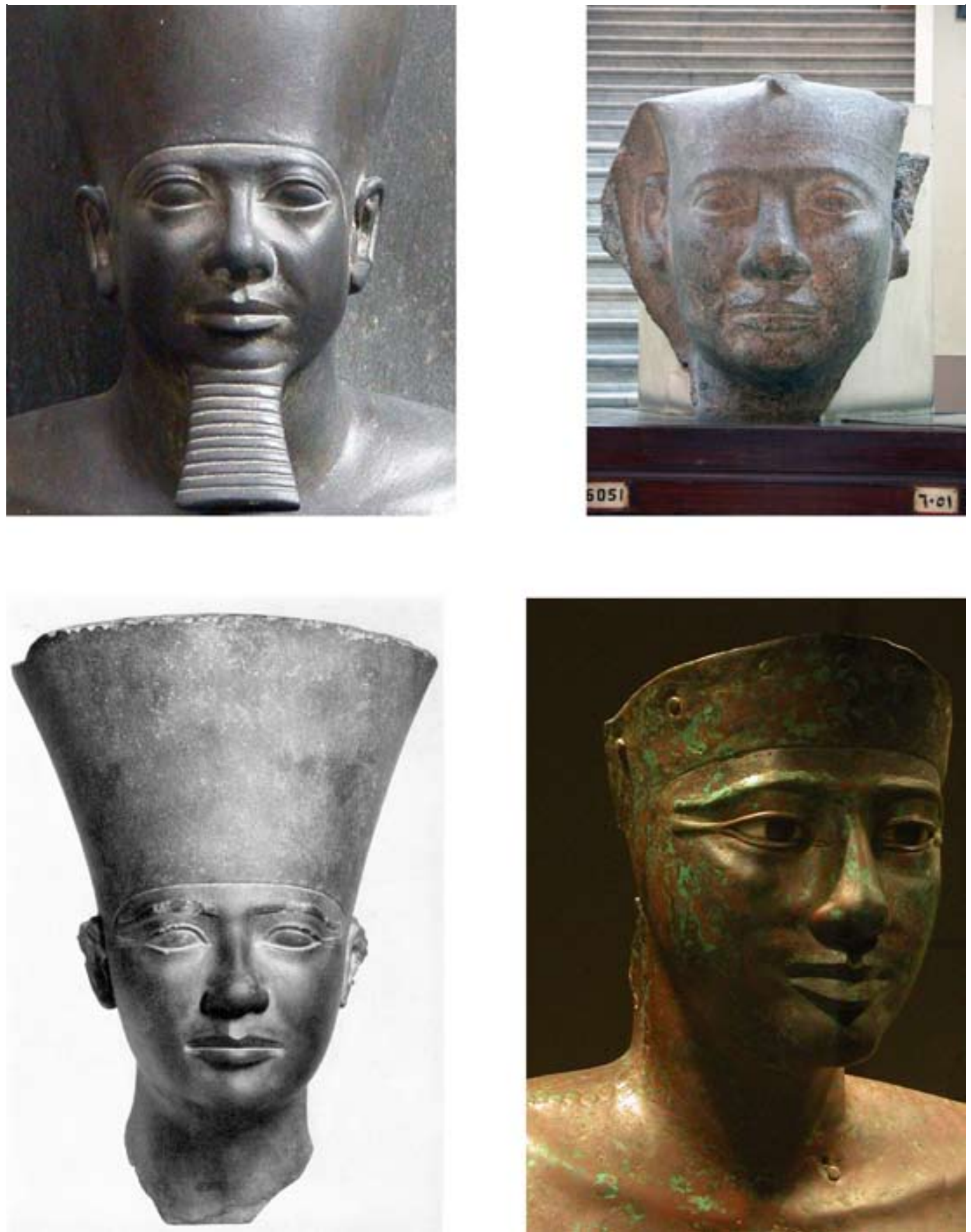

Pl. 4: Comparaison des caractéristiques physionomiques de Mykérinos (Le Caire JE 46499), d’Ouserkaf (Le Caire CG 52501 et JE 90220 [d'après Cl. Vandersleyen, Das alte Ägypten (Propyläen Kustgeschichte 15), Berlin, 1975, pl. 136b]) et de Pépi Ier (Le Caire JE 33034)(clichés de l'auteur). 
l'autre, quels que soient le matériau utilisé ou les dimensions de l'œuvre, et parce que, d'autre part, ses effigies présentent un visage clairement distinct de celui de son père, Khafrê,-connu aujourd'hui sous le nom hellénisé de Khéphren, - et de celui de son oncle, Radjédef, soit ses deux prédécesseurs immédiats (pl. 2). Ceci révèle assurément une individualisation volontaire et conséquente, même si le traitement plastique des yeux, des oreilles, de la bouche, etc, c'est-à-dire le vocabulaire stylistique de sa physionomie est tout à fait caractéristique des standards artistiques de la $\mathrm{IV}^{\mathrm{e}}$ dynastie, dont ce souverain fait partie $e^{29}$.

Les célèbres triades de Mykérinos, exhumées dans son temple funéraire à Guiza, sont d'un intérêt tout particulier pour l'étude du portrait dans l'art égyptien car, de manière assez exceptionnelle, chacune des ces sculptures présente trois visages: celui du monarque, celui de la déesse Hathor, qui l'accompagne et l'accueille, et celui de la figure d'un nome,- ou province d'Égypte,- qui symbolise l'apport d'offrandes de sa région ${ }^{30}$, chaque tête reproduisant, bien entendu, la physionomie royale (pl. 3). Comme leur découvreur, G. A. Reisner, l'avait judicieusement relevée ${ }^{31}$, chaque triade conservée se caractérise par de légères variations stylistiques, qui suffisent à la distinguer des autres et sont, en même temps, parfaitement constantes sur les trois visages d'une même œuvre, dénotant une seule et même main, ou plus exactement un seul et même artiste derrière (l'achèvement, au moins, de) chacune de ces sculptures, avec ses propres habitudes pour creuser un sillon entre la bouche et la joue, marquer les commissures et le rebord des lèvres ou souligner le contour des yeux. Combinée à la cohérence physionomique des visages sculptés du monarque, la nature et la distribution de ces différences stylistiques suggère l'existence d'un visage-modèle parfaitement contrôlé par le pouvoir central et diffusé dans les ateliers de production statuaire, où il était recopié le plus fidèlement possible, moyennant quelques petites altérations inhérentes aux facteurs humains et techniques qui interviennent nécessairement dans la réalisation de telles œuvres ${ }^{32}$. En plus de la recherche de cohérence physionomique, toute étude du portrait dans l'art égyptien doit donc prendre en considération cette inévitable variabilité.

Le portrait de Mykérinos est en outre intéressant dans la perspective qui nous occupe ici car, avec son nez très caractéristique et ses proportions faciales particulières, il exercera une influence considérable sur la représentation officielle de ses successeurs, comme, par exemple, Ouserkaf, premier roi de la $\mathrm{V}^{\mathrm{e}}$ dynastie, ou Pépy I ${ }^{\mathrm{er}}$, second souverain de la VI ${ }^{\mathrm{e}}$ dynastie, près de deux siècles plus tard (pl. 4$)^{33}$.

\section{Sésostris (Senousret) III et Amenemhat III}

La statuaire de Sésostris (en ancien égyptien Senousret) III et de son fils et successeur direct Amenemhat III constitue probablement l'un des sujets les plus discutés dans l'histoire du débat sur l'existence du portrait en Égypte antique. Elle illustre parfaitement les dangers et les difficultés

(29) À ce sujet, cf. Junge, dans Kunst des Alten Reiches (op. cit. supra, n. 8).

(30) Pour l'examen des relations établies entre les personnages de ces groupes statuaires et la manière dont elles sont signifiées, cf. LABOURY, «De la relation spatiale entre les personnages des groupes statuaires royaux en Égypte pharaonique», Revue d'Égyptologie 51 (2000), p. 65-83.

(31) Cf. G.A. Reisner, Mycerinus, the Temples of the Third Pyramid at Giza, Cambridge, Massachusetts., 1931, p. 127-129.

(32) Cf. Laboury, «M. Seidel, "Die königlichen Statuengruppen. Band I: Die Denkmäler vom Alten Reich bis zum Ende der 18. Dynastie" ", Bibliotheca Orientalis 57 (2000), col. 325-326.

(33) Outre les implications politiques que peut revêtir une telle revendication physionomique vis-à-vis du dernier des constructeurs des trois impressionnantes pyramides du plateau de Guiza, sur la "généralisation» ou la neutralisation des traits particuliers qui caractérise l'art du portrait durant la seconde moitié de l'Ancien Empire, cf. Assmann, dans Studies Simpson (op. cit. supra, n. 5), p. 65-67. 
d'interprétation engendrés par ce que Roland Tefnin a proposé d'appeler «le vertige du réalisme» ${ }^{34}$. L'individualisation extraordinaire qui semble caractériser les statues de ces deux pharaons (pl. 6a-b) a en effet, dès les débuts de l'égyptologie, fortement impressionné les commentateurs; et, à de rares exceptions près, ceux-ci ont tous été convaincus de la volonté de réalisme absolu qui aurait animé les sculpteurs de ces œuvres, légitimant de ce fait des lectures psychologiques de telles effigies, dont l'ambition aurait été d'exprimer la lassitude - voire la souffrance - royale après un long et fatiguant exercice du pouvoir. Selon cette interprétation très largement admise, telle une évidence, la variabilité stylistique que l'on constate dans les portraits de Sésostris III et d'Amenemhat III, comme dans l'iconographie de n'importe quel autre pharaon, - s'expliquerait par le vieillissement du souverain, scrupuleusement transposé, étape par étape, dans de nouvelles statues réalisées pour l'occasion, et par des traditions locales d'ateliers de sculptures, - une de ces hypothèses qui sévissent de longue date en histoire de l'art égyptien mais attendent toujours de pouvoir être démontrées, en tout cas pour l'art royal.

Ce modèle interprétatif, tout traditionnel qu'il soit devenu au fil du temps et de sa répétition dans les manuels, n'en est pas moins hautement discutable. Sans même évoquer sa frappante incompatibilité avec ce que l'on sait de la personnalité historique de Sésostris III et d'Amenemhat III, qui comptent indubitablement parmi les rois les plus forts et les plus puissants qui aient régné sur l'Égypte, des arguments du strict domaine de l'histoire de l'art permettent d'invalider un tel raisonnement culturo-centrique. Comme Roland Tefnin l'a parfaitement souligné, le contraste flagrant qui oppose un visage interprété comme âgé et fatigué à un corps de toute évidence ferme, jeune et puissant s'explique difficilement, surtout dans une représentation que l'on suppose hyperréaliste ${ }^{35}$. Par ailleurs, dans son étude minutieuse et exemplaire de l'ensemble du corpus de la statuaire de Sésostris III et de son fils, - soit 136 œuvres prises en compte, - Felicitas Polz a pu établir que les plus récentes statues datables d'Amenemhat III, - qui proviennent de son complexe funéraire à Hawara (comme, par exemple, Le Caire CG 385 [pl. 5a]), ainsi que du petit temple de Médinet Madi (Le Caire JE 66322 et Milan RAN 0.9.40001 [pl. 5b-c]), achevé par son successeur,- montrent la physionomie à l'apparence la moins âgée, comme si le roi parvenait à rajeunir avec le temps ${ }^{36}$. En outre, si aucune particularité typologique ou physionomique ne peut

(34) Cf. R. Tefnin, «Les yeux et les oreilles du roi», dans M. Broze et Ph. Talon (éd.), L'atelier de l'orfèvre. Mélanges offerts à Ph. Derchain, Louvain, 1992, p. 148-156.

(35) Cf. ID., op. cit., p. 151. Un cas tout à fait analogue est analysé dans le présent volume par Fr. Queyrel, à propos des portraits de Romains de l'époque républicaine sur l'île de Délos.

(36) Cf. F. Polz, «Die Bildnisse Sesostris' III. und Amenemhets III. Bemerkungen zur königlischen Rundplastik der späten 12. Dynastie», Mitteilungen des Deutschen Archäologischen Instituts, Abteilung Kairo 51 (1995), p. 227-254 (p. 250 pour la datation des statues citées ci-dessus). Cet article constitue en réalité une synthèse du Magisterarbeit présenté par F. Polz à la Fakultät für Orientalistik und Altertumswissenschaft der Universität Heidelberg en 1990, intitulé «Studien zur Porträtplastik Sesostris' III und Amenemhets III.», et dont elle a eu l'extrême amabilité de me transmettre une copie. Le processus de rajeunissement iconographique attesté dans le portrait d'Amenemhat III n'est pas sans évoquer le style de divinisation ou «Deification style» d'Amenhotep III (W. R. Johnson, «Monuments and Monumental Art under Amenhotep III. Evolution and Meaning», dans D. O'Connor et E. H. Cline [éd.], Amenhotep III. Perspectives on His Reign, Ann Arbor, 1998, p. 63-94; et infra), un pharaon qui semble précisément s'être inspiré du programme statuaire du complexe funéraire du fils de Sésostris III à Hawara, comme le suggère W. R. Johnson, "Amenhotep III and Amarna", Journal of Egyptian Archaeology 82 (1996), p. 68, n. 16. Deux groupes sculptés découverts sur ce site d'Hawara invitent d'ailleurs à penser qu'Amenemhat III,- comme Amenhotep III, son lointain successeur et émule, quatre siècles et demi plus tard (cf. infra),- se serait divinisé de son vivant, puisqu'ils figurent le souverain, dans l'attitude de l'adoration, en présence de son double,- avec l'uræus royal au front,- qui lui tend le signe de vie, comme le font les dieux dans l'iconographie égyptienne; cf. M. SEIDEL, Die königlichen Statuengruppen. Band I: Die Denkmäler vom Alten Reich bis zum Ende der 18. Dynastie (Hildesheimer Ägyptologische Beiträge 42), Hildesheim, 1996, p. 101-4, pl. 28. 

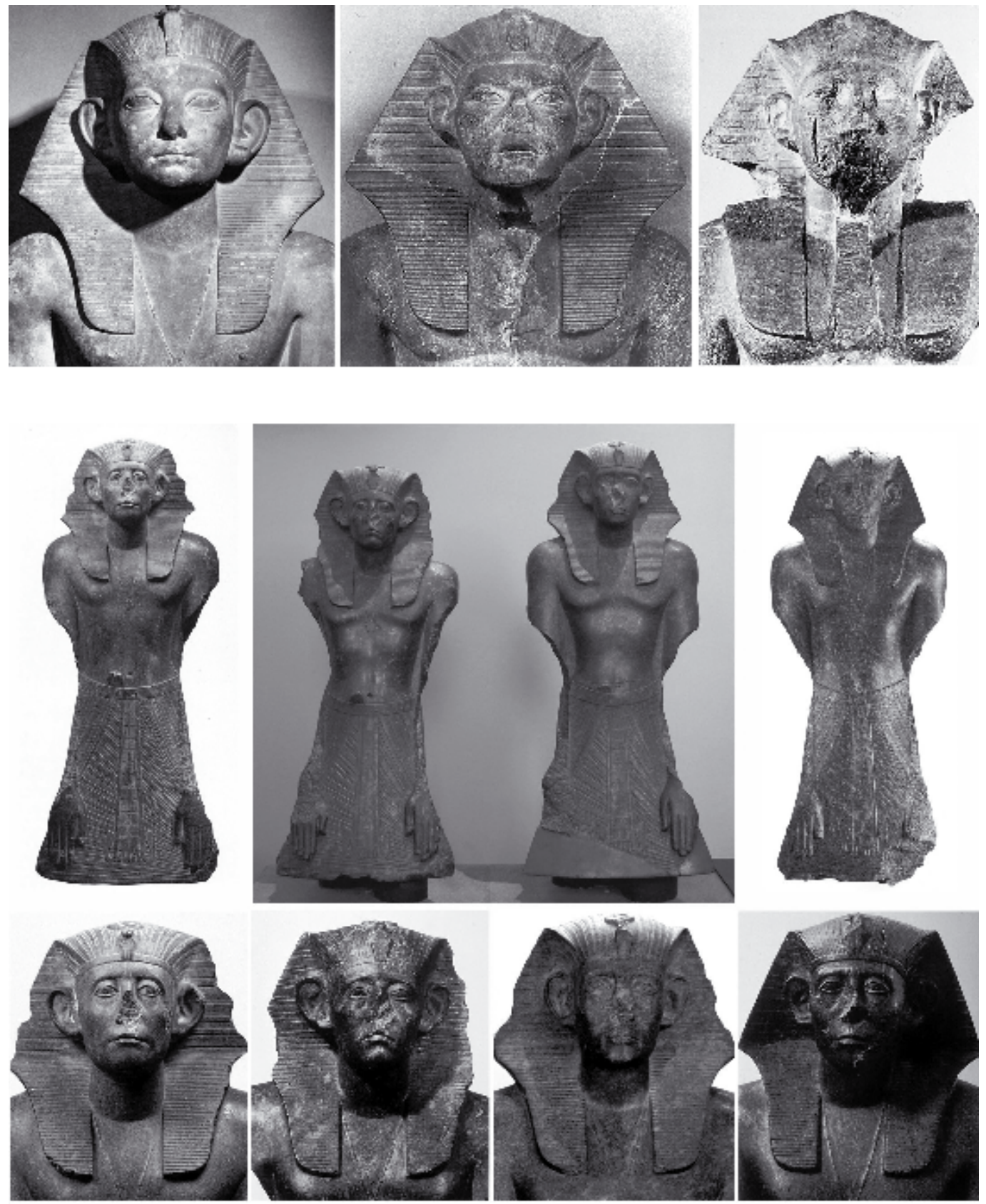

Pl. 5: a) statues tardives d'Amenemhat III: Le Caire CG 385, de Hawara (d'après D. WiLdung, L'âge d'or de l'Égypte. Le Moyen Empire, Paris, 1984, p. 207); et Le Caire JE 66322 et Milan RAN 0.9.40001, de Médinet Madi (d'après F. Polz, «Die Bildnisse Sesostris' III. und Amenemhets III. Bemerkungen zur königlischen Rundplastik der späten 12. Dynastie», Mitteilungen des Deutschen Archäologischen Instituts, Abteilung Kairo 51 [1995], pl. 52c-d);

b) variations des statues de Sésostris III au sein d'une même série: Le Caire RT 18-4-22-4, British Museum EA 686, 685 et 684, du temple de Montouhotep II à Deir el-Bahari (d'après M. SAleh et H. Sourouzian, Musée égyptien du Caire. Catalogue officiel, Mayence, 1987, n 98; Polz, op. cit., pl. 48a-b; E.R. Russmann, Eternal Egypt. Masterworks of Ancient Art from the British Museum, Berkeley, 2001, p. 103; et clichés de l'auteur). 
être mise exclusivement en relation avec un site ou une région donnés ${ }^{37}$, les statues qui proviennent d'une seule et même série présentent d'incontestables variations stylistiques dans la reproduction du visage-modèle du roi (pl. 5d), - exactement comme les triades de Mykérinos examinées précédemment. Enfin, l'émancipation vis-à-vis de la tendance traditionnelle à l'abstraction hiéroglyphique et la physionomie très marquée qui caractérisent véritablement les portraits de Sésostris III et d'Amenemhat III font, en réalité, leur apparition une génération plus tôt, dans la statuaire privée ${ }^{38}$ (pl. 6c), - qui, au moins dans ce cas particulier, semble avoir exercé une influence sur l'art royal.

Même si l'on admet l'idée d'une sorte d' «emprunt de personnalité» que suggère Fr. Junge dans ce contexte ${ }^{39}$, - un concept qui, à nouveau, tend à effacer l'opposition théorique entre portrait et image idéale ou image type, - les effigies de Sésostris III et d'Amenemhat III ne peuvent être envisagées comme l'expression d'un "amour du réalisme», qui, selon Jacques Vandier, auteur du célèbre Manuel d'archéologie égyptienne, aurait justifié "que de nouveaux portraits officiels aient été exécutés chaque fois que le roi changeait physiquement, dans un sens qui ne pouvait qu'être désagréable à l'amour-propre du souverain. $»^{40}$. De toute évidence, ces statues véhiculent un message à propos de la nature du pouvoir royal tel qu'il était conçu à l'époque, en résonance avec une très importante production littéraire contemporaine sur le même sujet, - notamment par le recours à des effets de réel appuyés, capables de suggérer des qualités particulières en relation avec la bouche, les yeux et les
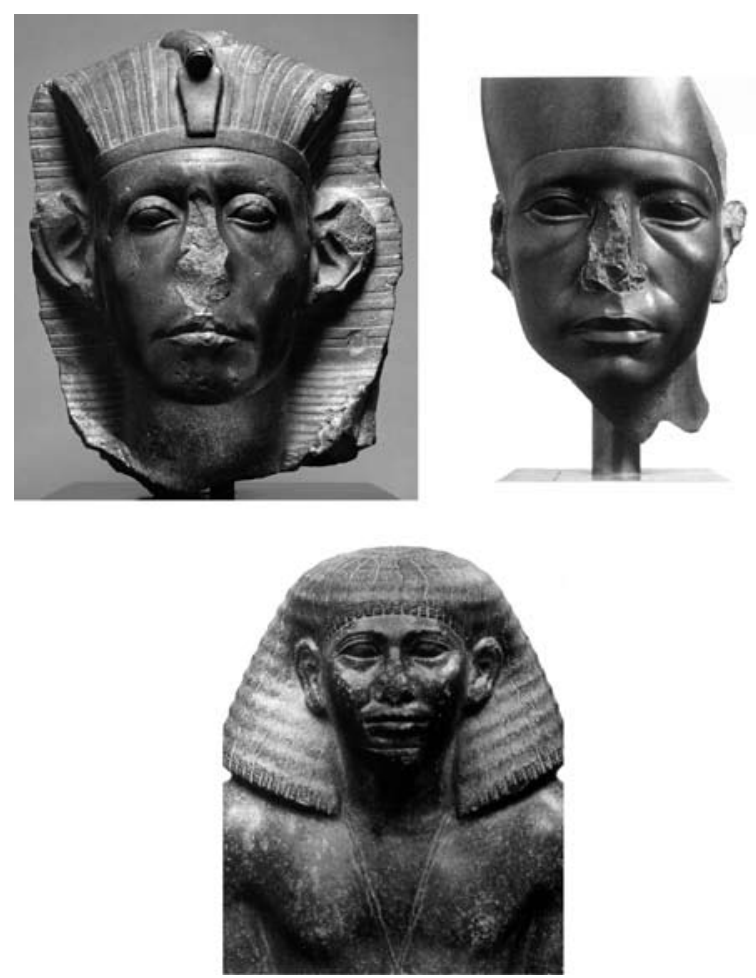

Pl. 6: tête de Sésostris III (Kansas City, William Rockhill Nelson Gallery of Art 62.11; d'après le site internet www.echoesofeternity. umkc.edu), tête d'Amenemhat III (Copenhague NYC AEIN 924; d'après Polz, op. cit., pl. 51c) et buste d'une statue du nomarque Sarenpout II (Éléphantine; règne d'Amenemhat II; d'après L. HaваснI, The Sanctuary of Heqaib [Archäologische Veroffentlichungen 33 - Elephantine IV], Mayence, 1985, pl. 33).

(37) Comme le relève F. Polz, ceci n'a en fait rien d'étonnant, puisque plusieurs textes de l'époque font état de la mobilité des œuvres d'art, ainsi que de celle des artistes eux-mêmes, comme, par exemple, la biographie de Sarenpout I ${ }^{\text {er }}$, nomarque d'Éléphantine sous le règne de Sésostris I ${ }^{\mathrm{er}}$,- soit un siècle plus tôt,- qui fit venir dans sa ville, en face de l'actuelle Assouan, une centaine d'artistes de la résidence royale, alors située à Lisht, près du Fayoum, à environ 800 kilomètres plus au nord; cf. Polz, op. cit., p. 205-1; L. Навасні, The Sanctuary of Heqaib (Archäologische Veroffentlichungen 33 - Elephantine IV), Mayence, 1985, p. 38.

(38) Cf. Fr. Junge, «Die Provinzialkunst des Mittleren Reiches in Elephantine», dans Habachi, op. cit., p. 117-139. Pour être précis, à cette époque, statuaire royale et statuaire privée semblent explorer de concert un rendu de type plus naturaliste de la physionomie humaine, mais, dans l'état actuel de nos connaissances, le portrait privé apparaît sur ce point en avance sur l'art royal, qui reste encore, à ce moment, plus traditionnel; à ce sujet, cf. B. FAY, The Louvre Sphinx and Royal Sculpture from the Reign of Amenemhat II, Mayence, 1996, p. 60 et pl. 80.

(39) Cf. ID., op. cit., p. 122.

(40) Cf. J. Vandier, Manuel d'archéologie égyptienne III, Paris, 1958, p. 194. 
oreilles, mais qui n'ont assurément rien à voir avec le concept occidental et moderne d'hyperréalisme ${ }^{41}$. Dans de telles conditions et en l'absence des momies de ces deux souverains, il est absolument impossible d'évaluer le degré de ressemblance entre le véritable visage du pharaon et ses portraits sculptés, même si une certaine convergence physionomique paraît assez vraisemblable, puisque la même formule stylistique fut déclinée différemment pour Sésostris et pour Amenemhat (pl. 6a-b).
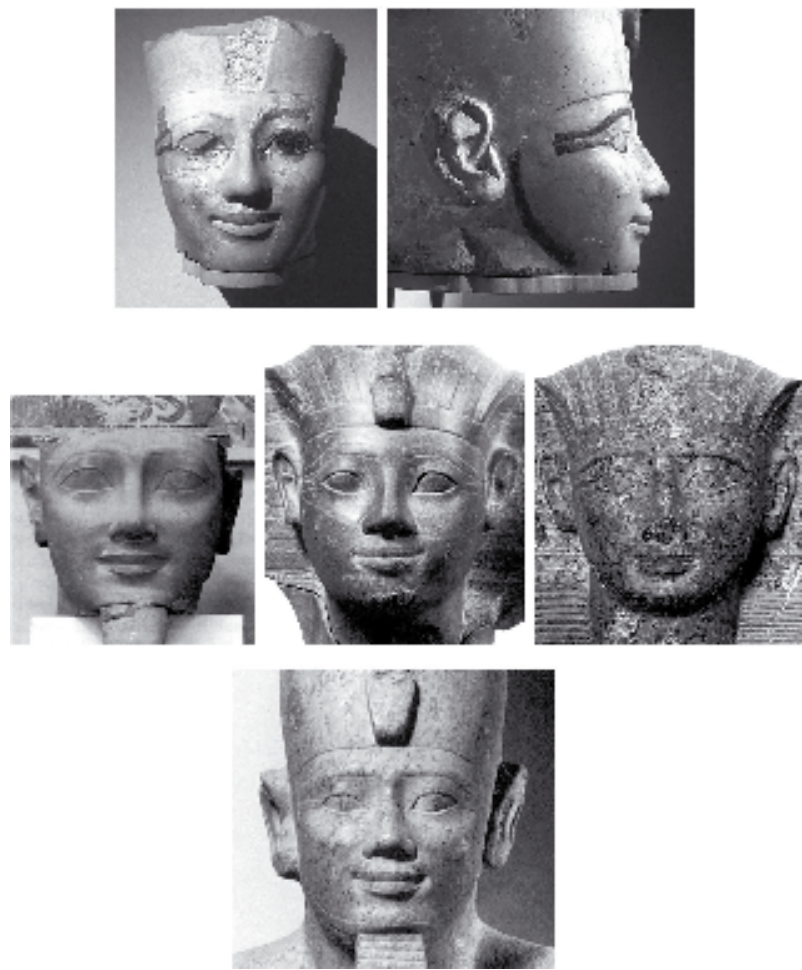

Pl. 7: tête de colosses osiriaques A de Hatshepsout à Deir el-Bahari (MMA 31.3.154-5; clichés de l'auteur), comparée à Le Caire CG 42051 (Thoutmosis Irr; cliché de l'auteur), JE 52364 (Thoutmosis II, d'après L. GABOLDE, «Indices nouveaux pour l'attribution de la tête JE 52364 à une statue de Thoutmosis II", Mitteilungen des Deutschen Archäologischen Instituts, Abteilung Kairo 56 [2000], pl. 23a), RT 14/6/24/11 (Thoutmosis III, période de la régence; cliché de l'auteur) et JE 38286 (Sésostris I Ir ; d'après H. SourouzIAN, «Standing royal colossi of the Middle Kingdom reused by Ramesses II», Mitteilungen des Deutschen Archäologischen Instituts, Abteilung Kairo 44 [1988], pl. 74)

\section{Hatshepsout}

L'évolution de l'image officielle du pharaon féminin Hatshepsout représente certainement l'illustration la plus évidente de l'écart que le portrait égyptien peut introduire vis-à-vis de l'apparence physique de son modèle.

Comme Roland Tefnin l'a démontré, cette évolution se déroule en trois phases principales ${ }^{42}$. Dans un premier temps, la reine, jusqu'alors régente de son neveu et beau-fils Thoutmosis III, décide d'assumer seule la royauté véritable et se fait représenter avec une titulature royale et les attributs traditionnels de pharaon, mais en tant que femme, c'est-à-dire avec une anatomie et des vêtements explicitement féminins. Dans cet esprit, le visage de ses statues n'est alors rien d'autre qu'une version féminisée, - soit peinte en jaune, couleur traditionnelle des femmes dans les conventions de l'art égyptien, - du portrait officiel de ses trois prédécesseurs directs,- son neveu et beau-fils, son époux et son père ${ }^{43}$, - portrait officiel lui-même directement inspiré de l'iconographie de Sésostris Irer, qui avait régné sur l'Égypte cinq siècles plus tôt (pl. 7) ${ }^{44}$. Cependant, assez rapidement, cette sorte de masque généalogique se mue

(41) Pour une discussion plus détaillée du sujet, reprenant les analyses précédentes de D. WiLdung (Sesostris und Amenemhet. Ägypten im Mittleren Reich, Munich, 1984), R. Tefnin (dans Mélanges offerts à Ph. Derchain [op. cit. supra, n. 34]), F. Polz (op. cit.) et J. Assmann (dans Studies Simpson [op. cit. supra, n. 5]), cf. Laboury, «Le portrait royal sous Sésostris III et Amenemhat III", Égypte, Afrique \& Orient 30 (Août 2003 - L'art du Moyen Empire), p. 55-64.

(42) Cf. R. Tefnin, La statuaire d'Hatchepsout. Portrait royal et politique sous la $18^{e}$ dynastie (Monumenta Agyptiaca 4), Bruxelles, 1979. Pour une réévaluation complète de la thèse de R. Tefnin après les avis critiques formulés par B. Letellier (dans Bibliotheca Orientalis 38 [1981], col. 305-8) et P.F. Dorman (The Monuments of Senenmut. Problems in Historical Methodology (Studies in Egyptology), Londres et New York, 1988, p. 41 et 112), cf. LABoury, La statuaire de Thoutmosis III (op. cit. supra, n. 8), p. 592-608.

(43) Cf. ID., op. cit., p. 604.

(44) Cf. ID., op. cit., p. 478-481. 
en un visage encore non attesté et très personnalisé, avec un plan facial nettement triangulaire, des yeux félins très allongés sous des sourcils haut arqués, une bouche étroite, resserrée aux commissures, et un nez ostensiblement busqué (pl. 8a). La souveraine met simultanément en évidence les insignes de son nouveau pouvoir royal, portant une coiffe némès de plus en plus ample et échangeant sa robe pour le pagne shendjyt des pharaons masculins, tandis que son anatomie ne devient plus qu'allusivement féminine (pl. 8b), avec des chairs cette fois peintes en orange (pl. 8a et $9 \mathrm{~b}$ ), - entre le jaune des femmes et l'ocre rouge des hommes. R. Tefnin l'a bien souligné, cette deuxième étape de l'évolution iconographique de Hatshepsout exprime clairement le désir que celle-ci a d'affirmer sa propre personnalité en tant que monarque. La métamorphose de la reine devenue pharaon ne s'arrête néanmoins pas là et, relativement vite, semble-t-il, elle aboutit à une image totalement masculine, dans laquelle Hatshepsout a définitivement abandonné sa féminité ${ }^{45}$, avec une musculature explicitement virile, une peau ocre rouge et une physionomie qui apparaît comme une synthèse entre ses deux précédents visages officiels (pl. 9), c'est-à-dire un compromis entre son portrait très individualisé de la phase intermédiaire, - vraisemblablement inspiré de ses traits véritables, - et l'iconographie commune à ses trois prédécesseurs masculins, y compris le jeune Thoutmosis III, avec lequel elle décide de partager le trône ${ }^{46}$.

Cette évolution, indubitablement motivée par le souci et le besoin de légitimité de Hatshepsout, constitue, bien évidemment, un cas extrême, dû à des circonstances politiques exceptionnelles, mais il démontre que même l'identité sexuelle peut être remodelée dans le portrait pharaonique, en fonction d'une image idéale ou idéelle, en l'occurrence celle du roi légitime de la tradition ${ }^{47}$. Concernant le rendu de sa physionomie, la souveraine offre un très bel exemple sinon d'emprunt de personnalité, au moins d'un emprunt partielle d'identité, en tant qu'héritière de certains prédécesseurs, intégrant dans son visage officiel des éléments faciaux reconnus comme les leurs, tels une sorte de signature physionomique de sa filiation, garantie et manifeste iconographique de sa légitimité.

\section{Thoutmosis (Thoutmose) III}

La problématique du portrait de Thoutmosis III est assez semblable et parallèle à celle de l'iconographie de Hatshepsout, faisant intervenir plusieurs phases successives, motivées par des revendications et réorientations politiques ${ }^{48}$, mais la momie du roi est cette fois bien conservée, et elle permet la comparaison entre le véritable visage du modèle et la physionomie qui lui est prêtée sur ses différentes statues ${ }^{49}$.

(45) Même si, dans les inscriptions de l'époque, les épithètes et les pronoms se rapportant à Hatshepsout peuvent encore, sporadiquement, comporter les marques du féminin, à partir du début de cette troisième phase, les images de la souveraine ne laissent plus paraître le moindre indice de sa féminité.

(46) Sur les relation iconographiques et politiques entre Hatshepsout et Thoutmosis III, cf. ID., op. cit.

(47) Hatshepsout est la seule souveraine d'Égypte qui ait éprouvé la nécessité de cette fiction iconographique qui consiste à se présenter comme un roi masculin (cf. E. STAEHELIN, "Zum Ornat an Statuen regierender Königinnen", Bulletin de la Société d'Égyptologie de Genève 13 [1989][Mélanges R. Hari], p. 145-156); pour l'interprétation de cette singularité, cf. LABOURY, op. cit., p. 628.

(48) Cf. Laboury, La statuaire de Thoutmosis III (op. cit. supra, n. 8). Une synthèse en anglais a été publiée dans ID., "Royal Portrait and Ideology. Evolution and Signification of the Statuary of Thutmose III», dans E. H. Cline et D. O'Connor (éd.), Thutmose III: A New Biography, Ann Arbor, 2006, p. 260-291, pl. 7.1-5. La qualité du corpus, avec 162 œuvres, plus de 25 sites d'origine différents et un très riche matériel de comparaison en deux dimensions, permet par ailleurs de véritablement démontrer ici le caractère exclusivement chronologique des différents types physionomiques attestés, et, par la même occasion, la centralisation au plus haut niveau du pouvoir des décisions qui motivaient les changements en matière d'iconographie royale.

(49) Pour un examen détaillé de ce qui suit, le lecteur pourra se reporter à ID., La statuaire de Thoutmosis III, p. 647-655. 
LE PORTRAIT ROYAL ET SON FONCTIONNEMENT DANS L'ÉGYPTE PHARAONIQUE
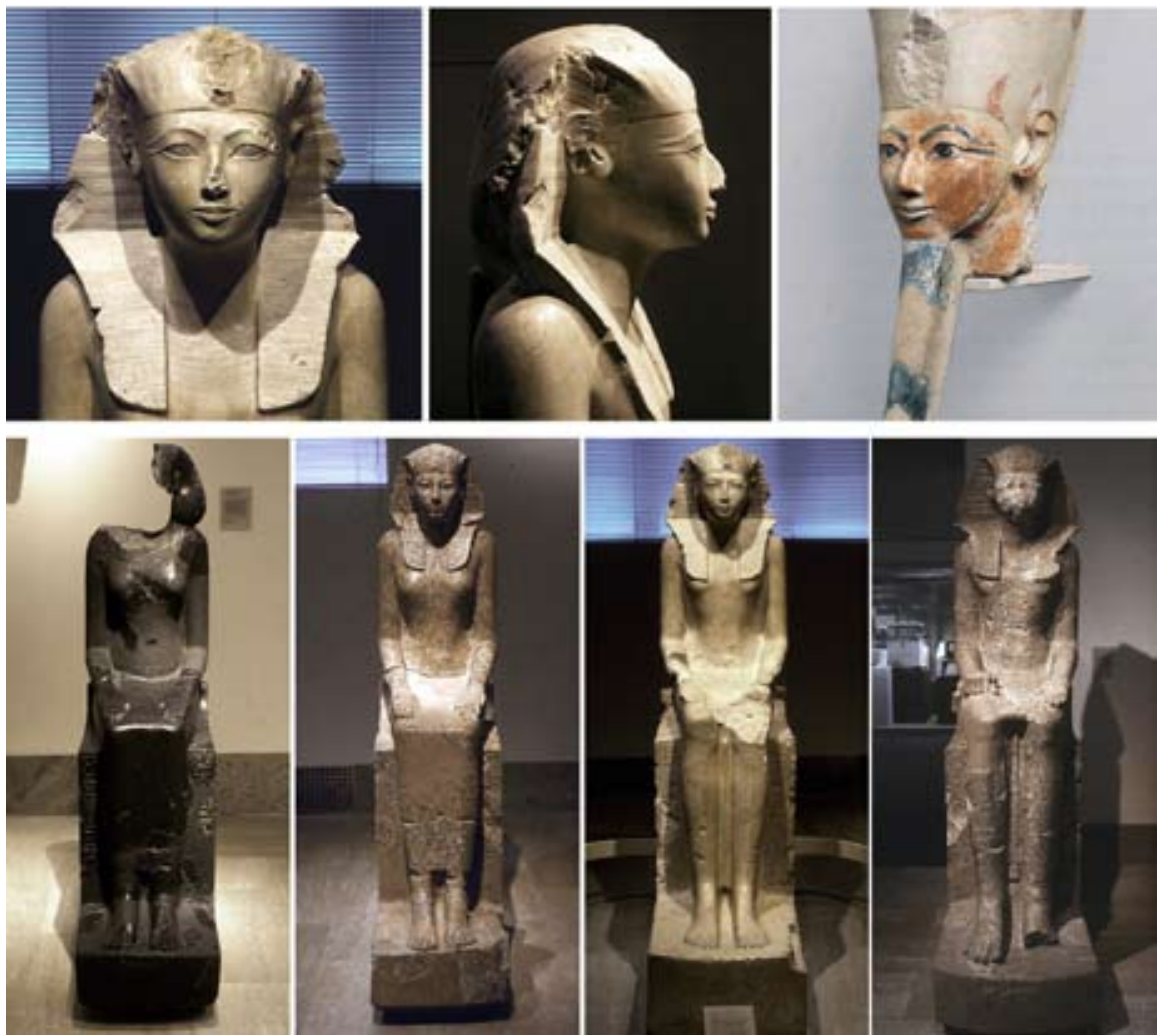

Pl. 8: a) MMA 29.3.2 (clichés de l'auteur) et tête du colosse osiriaque B MMA 31.3.164 (d'après C.H. RoeHRIG [éd.], Hatshepsut. From queen to pharaoh, New York, 2005, p. 140); b) statues assises de Hatshepsout provenant de Deir el-Bahari, dans l'ordre chronologique de leur réalisation: MMA 30.3.3, MMA 29.3.3, MMA 29.3.2 et MMA 27.3.164 (clic. de l'auteur).
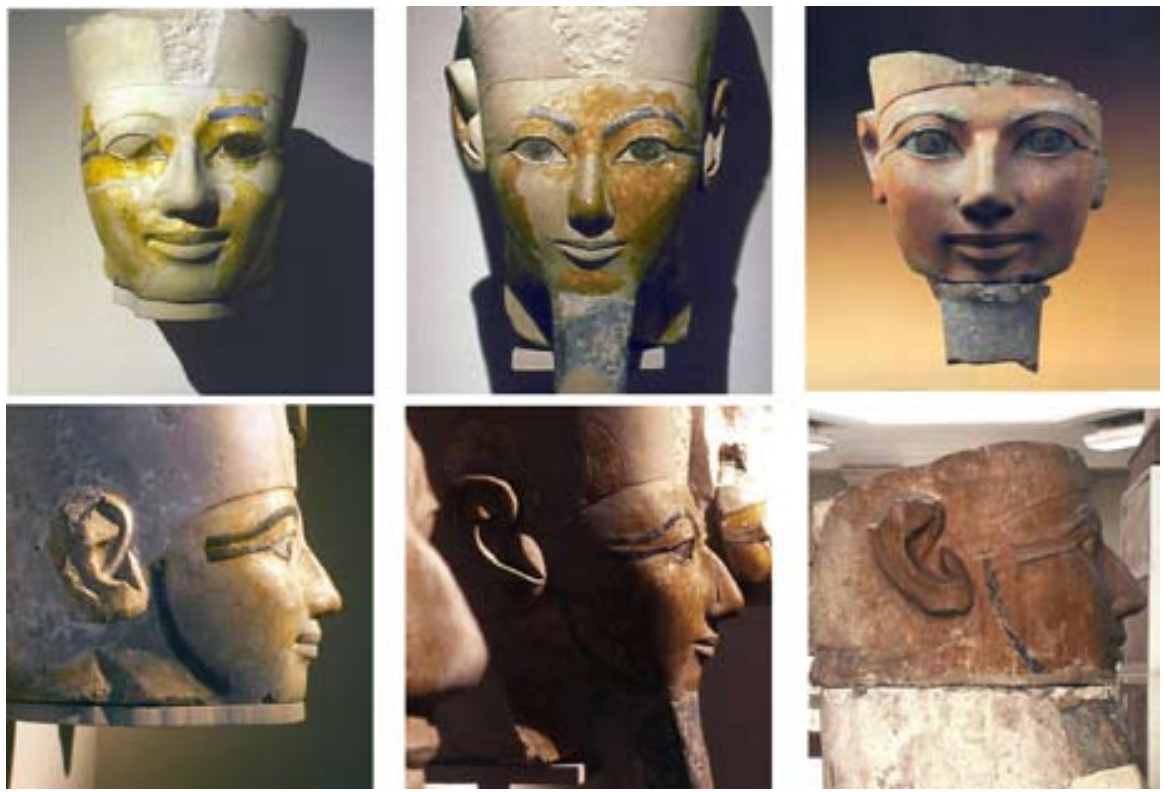

Pl. 9: les trois phases de l'évolution du portrait du pharaon Hatshepsout, illustrées par les colosses osiriaques A (MMA 31.3.154-5), B (MMA 27.3.163 [profil inversé]) et C (Le Caire JE 56259a-56262; d'après M. SALEH et H. Sourouzian, Musée égyptien du Caire. Catalogue officiel, Mayence, 1987, n 129 et clichés de l'auteur) de Deir el-Bahari. 


\section{DIMITRI LABOURY}
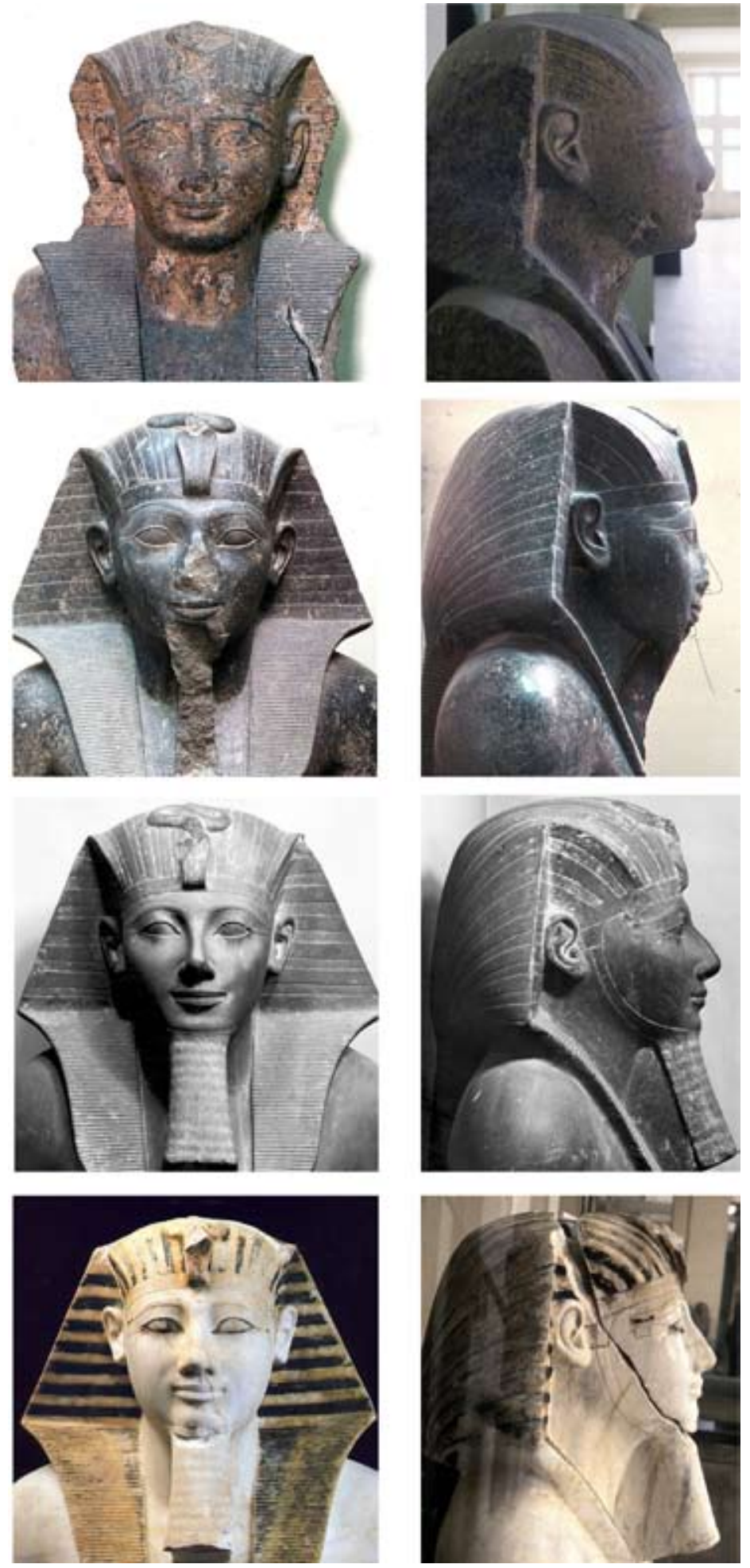

Pl. 10: les quatre phases principales de l'évolution du portrait de Thoutmosis III, illustrées par les statues Le Caire RT 14-6-24-11, CG 578, Louqsor J 2 et Le Caire JE 90237 MMA 07.230.3 (d'après D. LABOURY, La statuaire de Thoutmosis III. Essai d'interprétation d'un portrait royal dans son contexte historique [Egyptiaca Leodiensia 5], Liège, 1998, p. 641). 

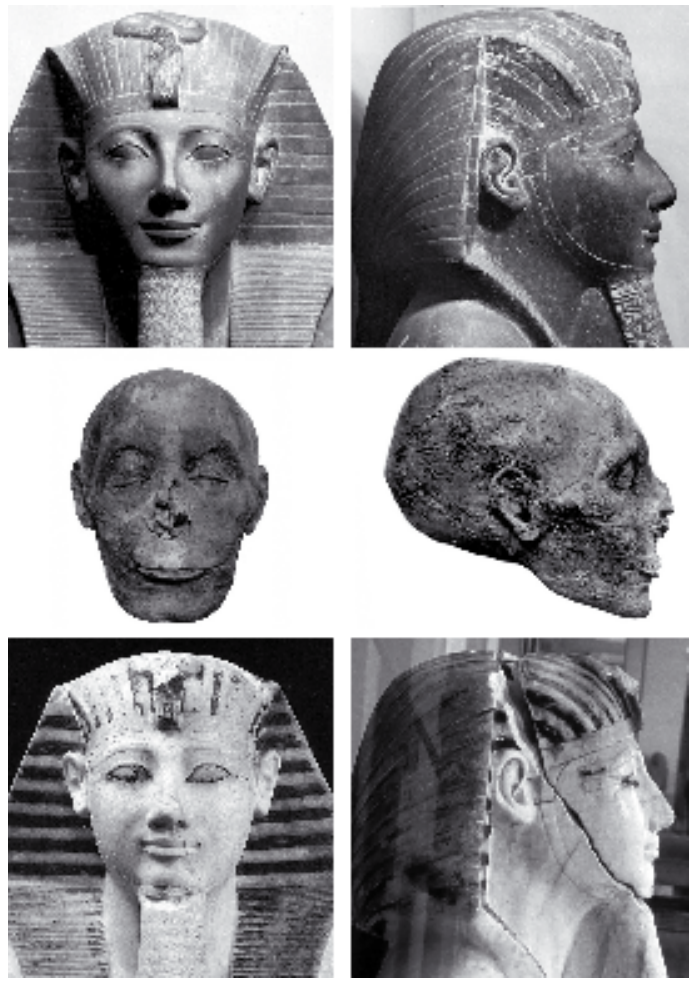

Pl. 11: comparaison entre le type physionomique des statues de Thoutmosis III au début de son règne autonome, la tête momifiée du roi et le type physionomique de ses statues durant la période de persécution de Hatshepsout (après l'an 42) (d'après Laboury, op. cit., p. 161-162, 339, 649, et cliché de l'auteur)

Malgré une évolution relativement importante à travers divers types chronologiques (pl. 10), l'iconographie de Thoutmosis III se caractérise par une série de particularités faciales absolument constantes, - une protubérance mentonnière au profil en forme de $S$, une mandibule importante et marquée, ainsi qu'une pommette basse et saillante, déterminant une dépression horizontale sous les yeux, - et qui se retrouvent précisément sur la momie du souverain (pl. 11), dénotant une indiscutable inspiration du visage réel du monarque. En revanche, d'autres détails physionomiques varient considérablement, se trouvant quelquefois en contradiction flagrante avec les traits de la momie: c'est notamment le cas, à la fin du règne, lorsque Thoutmosis III décrète la damnatio memoriae de Hatshepsout et décide de rectifier l'arête de son nez, - visiblement busquée sur sa momie, - afin d'éliminer le moindre élément de ressemblance avec sa tante et belle-mère, tout en calquant sa physionomie officielle sur celle de son père et de son grandpère, ses désormais seuls et uniques ancêtres légitimateurs (pl. 7). Cette variabilité et de tels emprunts vis-à-vis de l'iconographie de prédécesseurs plus ou moins directs montrent que des tendances esthétiques au réalisme ou à l'idéalisation ne peuvent suffire à expliquer l'évolution de la statuaire du roi. Il y a de toute évidence un décalage sciemment entretenu par rapport à l'apparence extérieure du modèle, qui permet d'introduire du sens et de signifier par des moyens physionomiques l'identité idéologique de l'individu portraituré.

Tout comme sa tante et belle-mère Hatshepsout, Thoutmosis III a suscité des modifications et, par là, une évolution de ses portraits parce que son identité, son auto-définition politique en tant que pharaon légitime, a changé au cours de son règne ${ }^{50}$. Ainsi, il semble clair qu'aux yeux des anciens Égyptiens, le portrait ne se contentait pas d'être une simple transposition plastique de l'apparence physique; il servait de définition figurative d'un individu et d'une identité reconnaissables, au-delà de cette apparence externe et même, si nécessaire, en dépit de celle-ci.

\section{Amenhotep III}

La statuaire d'Amenhotep III, arrière-petit-fils de Thoutmosis III, offre un autre exemple particulièrement intéressant d'émancipation signifiante vis-à-vis de l'apparence physionomique véritable du modèle portraituré.

(50) À ce sujet, cf. ID., op. cit., passim. 
Comme l'historien de l'art W. Raymond Johnson l'a parfaitement mis en évidence ${ }^{51}$, le début $\mathrm{du}$ fastueux règne d'Amenhotep III est caractérisé, suivant la tradition, par une continuation du modèle physionomique établi pour son père et prédécesseur direct, Thoutmosis IV (pl. 12a), avec néanmoins de très légers aménagements proportionnels qui semblent faire subtilement allusion au jeune âge du souverain à son avènement (très grands yeux, nez court, presque retroussé, ...). Durant les deux décennies qui suivent, l'art évolue, selon les tendances esthétiques de l'époque, vers un rendu toujours plus naturaliste des détails anatomiques, présentant l'effigie d'un souverain cette fois clairement mature (pl. 12b). Mais à l'abord de l'an 30, l'iconographie du monarque change très nettement, alors qu'il se prépare pour une fête que tout roi d'Égypte espérait pouvoir célébrer: le $\mathrm{Heb} \mathrm{Sed}$, un rituel qui remonte aux origines mêmes de la royauté pharaonique et avait pour but de renouveler l'autorité royale après un exercice de trente années, soit une génération. La célébration de la fête Sed d'Amenhotep III (il y en aura en réalité trois, aux changements des années 29-30, 33-34 et 37-38, peu de temps avant le décès du roi) est l'événement majeur du règne et donne lieu à des recherches très conséquentes dans le passé afin de réinventer ce rituel dans le contexte cosmopolite que connaît alors l'empire d'Égypte. À l'issue du Heb Sed, le roi s'en trouve non seulement rajeuni, comme s'il recommençait un tout nouveau règne, mais aussi divinisé, c'està-dire perçu idéologiquement comme une manifestation terrestre, tangible, de la divinité suprême, de nature fondamentalement solaire, dont il est, plus que jamais, l'enfant sur terre. W. R. Johnson a montré que cette nouvelle conception idéologique du souverain se traduit par l'apparition d'un nouveau style, qu'il propose de baptiser le «deification style». La parure d'Amenhotep III s'enrichit en effet ostensiblement d'attributs qui soulignent sa solarisation divinisante (tels de nombreux urcei coiffés d'un disque solaire) (pl. 12c); mais, surtout, dans la perspective qui nous occupe ici, sa physionomie est désormais traitée de façon beaucoup plus stylisée, avec de très grands yeux en amandes, d'apparence surnaturelle, et des proportions générales qui évoquent - à nouveau - les caractéristiques de l'enfance ( $\mathrm{pl} .12 \mathrm{~d}$ ), au point que l'un des commentateurs de la démonstration de Johnson ait proposé de parler de «la seconde jeunesse d'Amenhotep III ${ }^{52}$, une fiction iconographique qui manifeste la renaissance solaire du pharaon à l'occasion de sa fête Sed.

Outre que le portrait du roi décrit, une fois de plus, une évolution contraire à la nature, en l'occurrence celle d'un souverain qui rajeunit avec le temps ${ }^{53}$, on constate ici un usage sémiotique non plus d'effets de réel, mais bien d'effets d'irréalité, avec une stylisation très marquée, apte à suggérer la distance et la différence d'essence entre le personnage portraituré, qui relève du monde des dieux, et le commun des mortels.

\section{Le portrait royal dans l'atelier du sculpteur Thoutmose à Amarna}

L'exploration archéologique de l'atelier de sculpture mis au jour dans la propriété du «favori du dieu parfait (= le roi), l'artiste et sculpteur en chef Thoutmose» à Akhet-Aton - Amarna, - le site de l'éphémère capitale du pharaon monothéiste Amenhotep IV - Akhénaton,-- permet d'appréhender les modalités pratiques de création d'un portrait royal dans l'Égypte ancienne ${ }^{54}$.

(51) Pour l'étude de l'évolution de la statuaire d'Amenhotep III, cf. W. R. Johnson, «Images of Amenhotep III in Thebes: Styles and Intentions», dans L. M. Berman (éd.), The Art of Amenhotep III: Art Historical Analysis, Cleveland, 1990, p. 26-46; ID., «Monuments and Monumental Art under Amenhotep III» (op. cit. supra, n. 36).

(52) Cf. Cl. Vandersleyen, «Les deux jeunesses d’Amenhotep III», Bulletin de la Société Française d'Égyptologie 111 (avril 1988), p. 9-30.

(53) Cf. supra, le portrait d'Amenemhat III et les commentaires développés à la n. 36 sur le parallélisme que l'on peut établir entre les deux cas.

(54) Pour le détail de l’analyse évoquée ici, le lecteur pourra se reporter à D. LABoury, «Dans l'atelier du sculpteur Thoutmose», dans C. Cannuyer (éd.), La langue dans tous ses états. Michel Malaise in honorem (Acta Orientalia 18), Bruxelles / Liège / Louvain-la-Neuve, p. 289-300. 

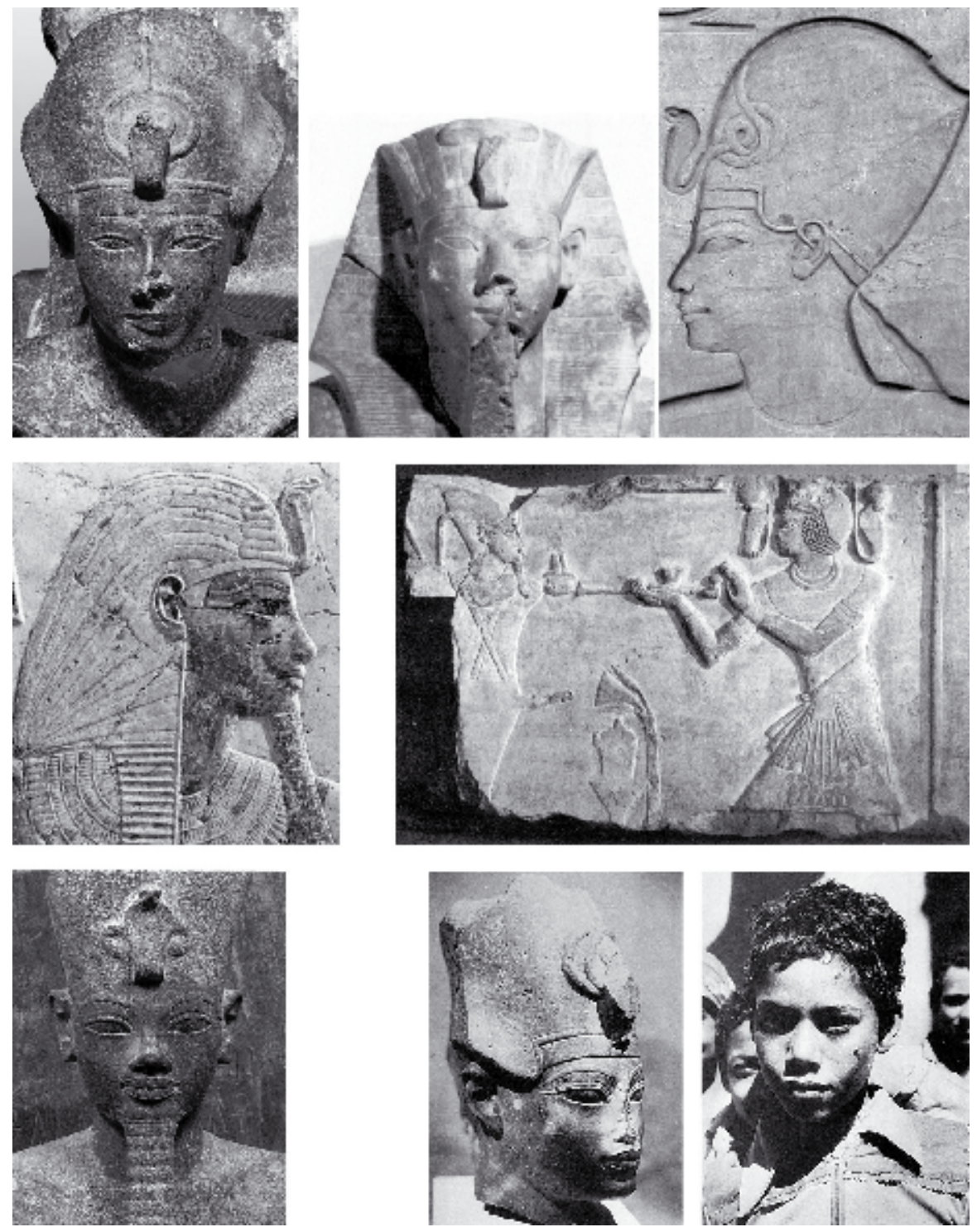

Pl. 12: a) statue d'Amenhotep III datant du début de son règne (Louqsor J 1006-1032; d'après H. Sourouzian, «Raccords de statues d'Aménophis III (suite)", Bulletin de l'Institut Français d'Archéologie Orientale 107 [2007], p. 238, fig. 21), comparée à une statue (Le Caire JE 43611; d'après B.M. BRYAN, «Portrait Sculpture of Thutmose IV », Journal of the American Research Center in Egypt 24 [1987], p. 15, fig. 21) et à un relief figurant son père, Thoutmosis IV (cour de fêtes édifiée par ce roi à Karnak; cliché de l'auteur);

b) Relief d'Amenhotep III dans le style de la décennie qui précède le $1^{\text {er }}$ Heb Sed (d'après S. BickeL, Tore und andere wiederverwendete Bauteile Amenophis' III. Untersuchungen im Totentempel des Merenptah in Theben III [Beiträge zur ägyptischen Bauforschung und Altertumskunde 16), Stuttgart, 1997, pl. 63);

c) bloc d'un monument d'Amenhotep III décoré dans le "Deification style» (Louqsor, Musée d'Art égyptien, J 45; d'après A.P. Kozloff, B.M. Bryan, L.M Berman et É. Delange, Aménophis III. Le Pharaon-Soleil, Paris, 1993, p. 83);

d) statue d'Amenhotep III réalisée dans le «Deification style» (Louqsor J 838; d'après M. EL-SAGHIR, Das Statuenversteck im Luxortempel, Mayence, 1992, p. 21) et comparaison proposée par K. Mysliwiec entre la tête MMA 56.138, de la même phase stylistique, et la tête d'un jeune garçon de Balat (d'après Mrsıiwiec, «The Art of Amenhotep III: A Link in a Continuous Evolution», dans L.M. Berman [éd.], The Art of Amenhotep III: Art Historical Analysis, Cleveland, 1990, pl. 9). 
Lomaine de "liftistc et 3c.lpteur en hel

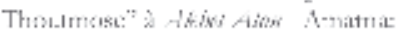

1 - minses de ithoumose

2 - piéce où trasut decouverts les modeles ct érudes de téres er de wikages en plárte- dont.

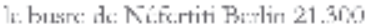

3 - ancler de plarrenc

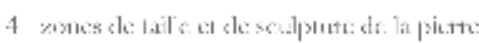

5-zones destinces ì soxuellir les ouvrers jecunabers de lacelier de sculpoure
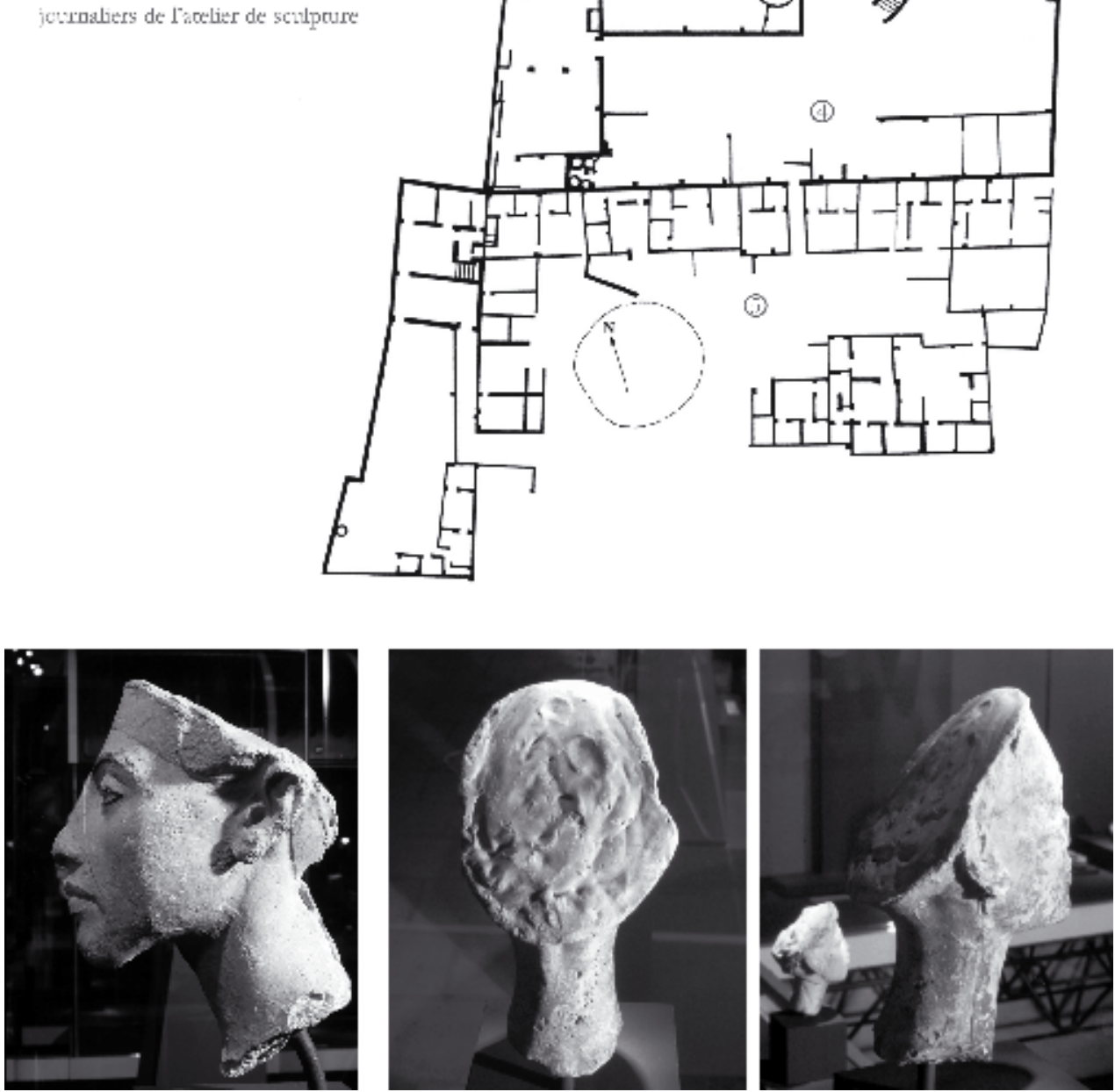

Pl. 13: a) plan du domaine de «l'artiste et sculpteur en chef Thoutmose» à Akhet-Aton - Amarna (d'après D. Laboury, "Dans l'atelier du sculpteur Thoutmose», dans C. CANnuyer [éd.], La langue dans tous ses états. Michel Malaise in honorem [Acta Orientalia 18], Bruxelles/ Liège / Louvain-la-Neuve, p. 290);

b) traces de modelage sur le tour de tête d'Akhénaton Berlin 21.351 (clichés de l'auteur);

c) traces de moulage sur le tour de tête de Néfertiti Berlin 21.349 (clichés de l'auteur). 
C'est là que le mondialement célèbre buste de Néfertiti (Berlin, Ägyptisches Museum 21.300) fut exhumé, dans une petite pièce attenante à l'antichambre de la maison proprement dite de Thoutmose et exclusivement accessible par cette antichambre, soit dans la partie domestique et privée du domaine du sculpteur (pl. 13a). La disposition archéologique des lieux invite à penser que cette pièce était aménagée pour ranger (et peut-être exposer) les objets qui y furent découverts ${ }^{55}$. Le buste de la reine s'y trouvait effectivement avec toute une série d'études de têtes et de visages en plâtre, qui matérialisent en fait les étapes successives de conception de l'image officielle d'un individu,- royal ou non. Ces plâtres présentent des traces matérielles de moulage (pl. 13c) et de modelage (pl. 13b), ce qui signifie qu'ils procèdent d'œuvres réalisées en matériau meuble, - vraisemblablement de l'argile, - puis moulées, afin d'être reproduites et figées en une épreuve en plâtre. Un tel procédé de pérennisation de l'esquisse en matériau malléable et le fait que la plupart de ces objets comportent encore des indications peintes ou incisées en vue de remaniement ou d'achèvement (pl. 13b) montrent que les étapes qu'ils représentent étaient sans doute induites par la nécessité d'un contrôle, très certainement exercé par - ou, en tout cas, en accord avec - le commanditaire de la future œuvre sculptée ${ }^{56}$. Si seulement deux étapes sont attestées pour le portrait privé (un masque, puis un visage, avec le début du cou, les oreilles et une ébauche de coiffure) (pl. 14), les effigies officielles des membres de la famille royale faisaient intervenir un processus de production plus complexe (pl. 14), en quatre phases successives, avec au moins trois paliers de contrôle avant la réalisation du modèle final: un buste sculpté en pierre, complété, voire corrigé, par des ajouts de plâtre et parachevé à l'aide de délicates peintures, d'incrustations précieuses ou même de dorures. Ces bustes de grande valeur pouvaient ensuite être dupliqués ${ }^{57}$ et envoyés dans les différents ateliers de sculpture de l'empire afin d'assurer l'uniformité de reproduction des traits du roi et de la reine sur leurs diverses statues.

En cherchant à aborder la question de la fascinante impression de beauté et de perfection qui se dégage du buste de Néfertiti, Rolf Krauss a reconstitué la vision originale du sculpteur en appliquant sur un relevé photogrammétrique, - donc parfaitement objectif et sans déformation perspective, de ce buste une grille graduée en unités métriques de l'époque, soit en doigts égyptiens $(1,875 \mathrm{~cm})$ (pl. 15a), suivant en cela le processus de conception des statues tel que le pratiquaient les artistes pharaoniques sur le bloc parallélépipédique à tailler $(\mathrm{pl} .15 \mathrm{~b})^{58}$. Il est ainsi apparu que chacun des traits déterminants du visage se trouve sur une ligne ou à une intersection de deux lignes de ce quadrillage, démontrant combien ce portrait qui passe généralement pour ce que l'art égyptien a produit de plus réaliste est artificiellement construit. En outre, suivant le même principe d'analyse, R. Krauss a également mis en évidence que la partie supérieure du visage d'Akhénaton et de Néfertiti,

(55) Cf. ID., op. cit., p. 291.

(56) Ces diverses opérations de modelage, de moulage et de corrections des esquisses étaient probablement assurées par Thoutmose lui-même, ou l'un de ses plus proches collaborateurs, dans la mesure où l'atelier de plâtrerie, - identifié par la présence de nombreuses éclaboussures de plâtre durcies, - n'était pas situé dans la zone de sculpture sur pierre, ouverte à des travailleurs journaliers dans la cour à l'arrière de la propriété, mais bien juste à côté de l'habitation privée du sculpteur en chef du roi, exclusivement accessible depuis celle-ci (pl. 13a); cf. J. Phillips, «Sculpture Ateliers of Akhetaten. An examination of two studio-complexes in the City of the Sun-Disk», Amarna Letters 1 (1991), p. 31-40; Laboury, op. cit., p. 296. L'étude des traces technologiques que portent ces esquisses en plâtre permet même de reconstituer la position précise dans laquelle l'artiste travaillait son modèle en matériau meuble: assis sur un siège plus bas que ses genoux (comme le montrent d'ailleurs les représentations d'ateliers de sculpteurs de l'époque), montant son œuvre sur une pièce de bois posée sur ses cuisses; cf. ID., op. cit., p. 298-300.

(57) C’est assurément le cas pour le buste d'Akhénaton, dont on possède deux exemplaires, - celui du Louvre, E 11076, et celui de l’Ägyptisches Museum de Berlin, inv. 21.360, - de dimensions pratiquement identiques (pl. 14).

(58) Cf. R. Krauss, «1913-1988. 75 Jahre Büste der NofretEte/ Nefret-iti in Berlin. Zweiter Teil. », Jahrbuch Preußischer Kulturbesitz 28 (1991), p. 123-157; ID., "Nefertiti - A Drawing-board of Beauty? The "most lifelike of Egyptian art" is Simply the Embodiment of Numerical Order», Amarna Letters 1 (1991), p. 46-49. 


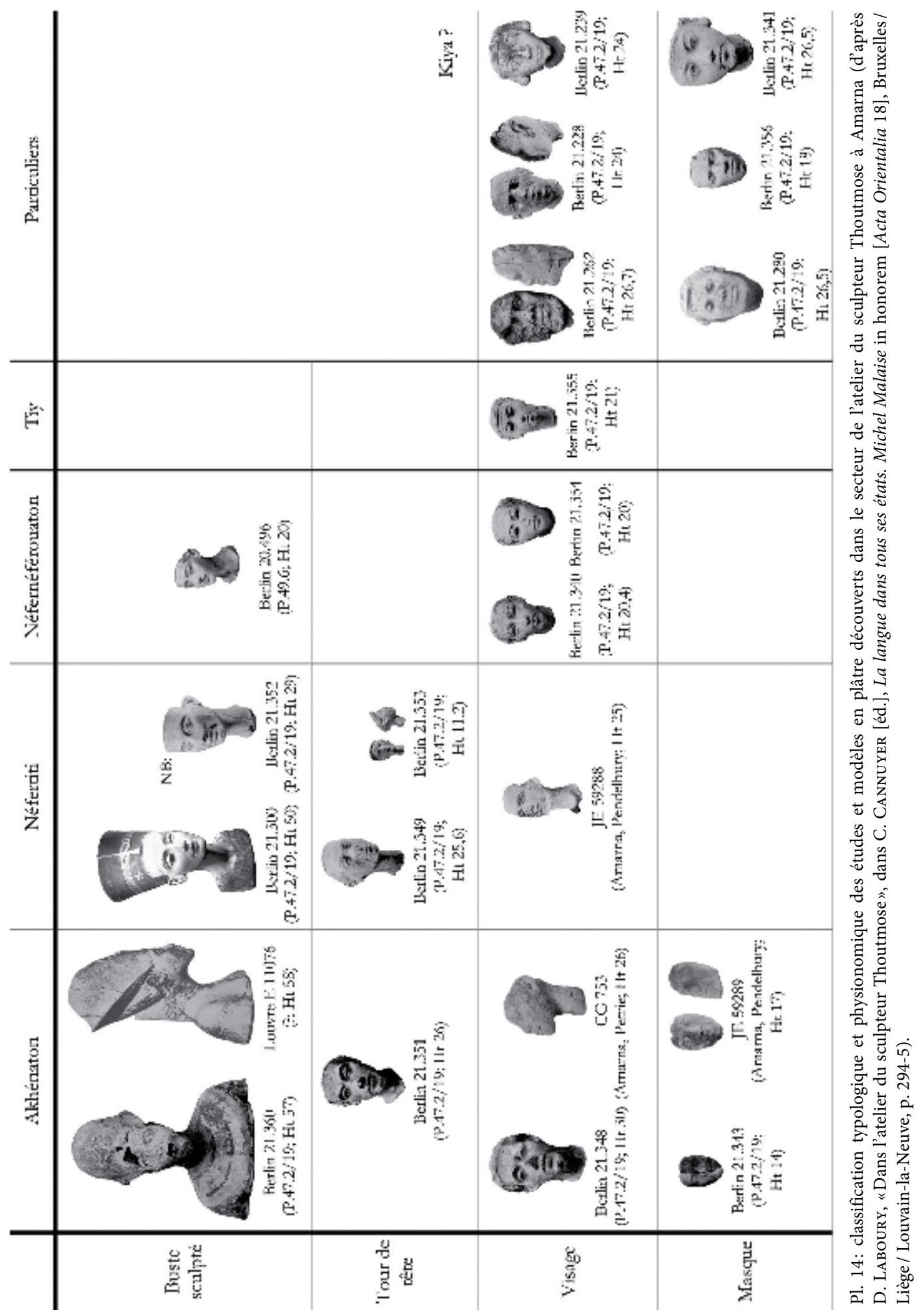


depuis la base du nez jusqu'au départ de la coiffure sur le front, est exactement identique, tant en dimensions qu'en morphologie (pl. 16a ${ }^{59}$. Dans ces conditions, même s'il est tentant d'imaginer une certaine convergence entre le visage véritable d'Akhénaton et de Néfertiti et leurs portraits sculptés, en apparence si individualisés, force est de conclure que leur image officielle les a, sans nul doute possible, idéalisés. La sublime Néfertiti, qui, dès sa révélation au grand public au début des années 1920, avait conquis le monde de la mode, puis celui de la publicité, incarne d'ailleurs l'idéal de beauté féminine de son temps, tel qu'on peut, notamment, l'appréhender à travers les textes de la poésie amoureuse du Nouvel Empire ${ }^{60}$.

Dans la perspective de ces portraits officiels qui résultent de véritables constructions plastiques, et dans le contexte politique de la fin de la XVIII ${ }^{e}$ dynastie $^{61}$, il n'est pas sans intérêt de constater que les effigies des deux successeurs directs d'Akhénaton, Néfernéférouaton ${ }^{62}$ et Toutankhamon, probablement deux enfants du couple royal atoniste ${ }^{63}$, apparaissent comme le produit d'une combinaison de traits physionomiques reconnus comme ceux d'Akhénaton et de Néfertiti (pl. 16a). Ressemblances familiales dans des portraits fidèles ou signification idéologique pour des images idéalisées? En ce qui concerne Toutankhamon, la conservation de sa momie permet en tout cas, à nouveau, de montrer que les statues du jeune souverain ne reproduisent pas exactement la physionomie véritable de leur modèle, mais offrent plutôt une image compatible avec celle-ci, et avec son jeune âge (pl. 16b), même si le visage de ce roi adolescent sera réutilisé comme une sorte de masque de légitimité par les trois pharaons qui lui succèderont, Ay, Horemheb et Ramsès Ier ( $\mathrm{pl} .16 \mathrm{c}$ ), qui, tous, sans exception, accédèrent au trône d'Égypte après une longue carrière civile ${ }^{64}$.

(59) Cf. ID., «Les représentations de Néfertiti et Akhénaton sont-elles réalistes?», dans Akhénaton et l'époque amarnienne (Bibliothèque d'Égypte, Afrique \& Orient), Paris, 2005, p. 135-144.

(60) Cf. J. Assmann, «Ikonographie der Schönheit im alten Ägypten», dans Th. Stemmler (éd.), Schöne Frauen Schöne Männer. Literarische Schöneheitsbeschreibungen. 2. Kolloquium der Forschungsstelle für europäische Literatur des Mittelalters (Forschungsstelle für europäische Lyrik des Mittelalters an der Universität Mannheim), Mannheim, 1988, p. 13-30; LABoury, "La beauté dans l'art du règne d'Amenhotep IV - Akhénaton», dans E. WARmenbol (dir.), Beautés d'Égypte. "Celles que les ans ne peuvent moissonner". Catalogue de l'exposition créée au Musée du Malgré-Tout à Treignes (Belgique) du 2 juin au 15 décembre 2002, Brugge, 2002, p. 51-8.

(61) Cf. M. Gabolde, D'Akhénaton à Toutânkhamon (Collection de l'Institut d'Archéologie et d'Histoire de l'Antiquité. Université Lumière - Lyon 2 3), Lyon, 1998; R. Krauss, «Eine Regentin, ein König und eine Königin zwischen dem Tod von Achenaten und der Thronbesteigung von Tutanchaten », Altorientalische Forschungen 34, 2 (2007), p. 294-318.

(62) Pour l'iconographie de ce pharaon mal connu, cf. LABOURY, « Mise au point sur l'iconographie de Néfernéférouaton, le prédécesseur de Toutankhamon", dans M. Eldamaty et May Trad (éd.), Egyptian Museum Collections around the World. Studies for the Centennial of the Egyptian Museum, Cairo II, Le Caire, 2002, p. 711-722

(63) Cf. supra, n. 61.

(64) Le phénomène se prolonge en réalité assurément jusqu'au début du règne de Séthi Irer, le fils et successeur de Ramsès Ir (cf. H. Sourouzian, «Statues et représentations de statues royales sous Séthi I ${ }^{\mathrm{er}}$ ", Mitteilungen des Deutschen Archäologischen Instituts, Abteilung Kairo 49 [1993], p. 239-257; P. J. Brand, The Monuments of Seti I. Epigraphic, Historical and Art Historical Analysis [Problem der Ägyptologie 16], Leyde/ Boston/ Cologne, 2000, p. 3 sq.). Avec le lancement de cette nouvelle dynastie, - la XIX'e, - ce souverain, qui est donc le premier roi d'Égypte après Toutankhamon dont la légitimité soit assurée par un père couronné pharaon, s'émancipe, après seulement quelques années de règne (ID., op. cit., p. 38), du modèle du dernier descendant de la prestigieuse XVIII ${ }^{e}$ dynastie. Il se présente d'ailleurs ostensiblement comme le restaurateur de l'ordre après les troubles de l'épisode de l'hérésie atoniste,- sous Akhénaton et ses successeurs immédiats (ID., op. cit., passim),- et apparait alors avec un visage plus allongé que celui de Toutankhamon et un nez qui se busque à nouveau, selon une physionomie qui servira de base au portrait officiel de son propre fils et successeur, Ramsès II. On a souvent considéré le visage de cette seconde iconographie de Séthi Ir comme une transposition fidèle des traits véritables de sa momie, admirablement conservée (ce qui a parfois pu influencer une telle lecture); cf. Spanel, Through Ancient Eyes. (op. cit. supra, n. 3), p. 2-3. Cependant, - une fois de plus, - on ne peut exclure la possibilité d'une inspiration vis-à-vis d'un autre illustre prédécesseur de la XVIII ${ }^{e}$ dynastie, en l'occurrence le grand conquérant Thoutmosis III (cf. supra), que Séthi I ${ }^{\text {er }}$ et ses descendants admiraient visiblement (cf. N. GRIMAL, Les termes de la propagande royale égyptienne de la XIXe dynastie à la conquête d'Alexandre [Études sur la propagande royale égyptienne 4 - Mémoires de l'Académie des Inscriptions et Belles-Lettres. Nouvelle Série 6], Paris, 1986, passim). 

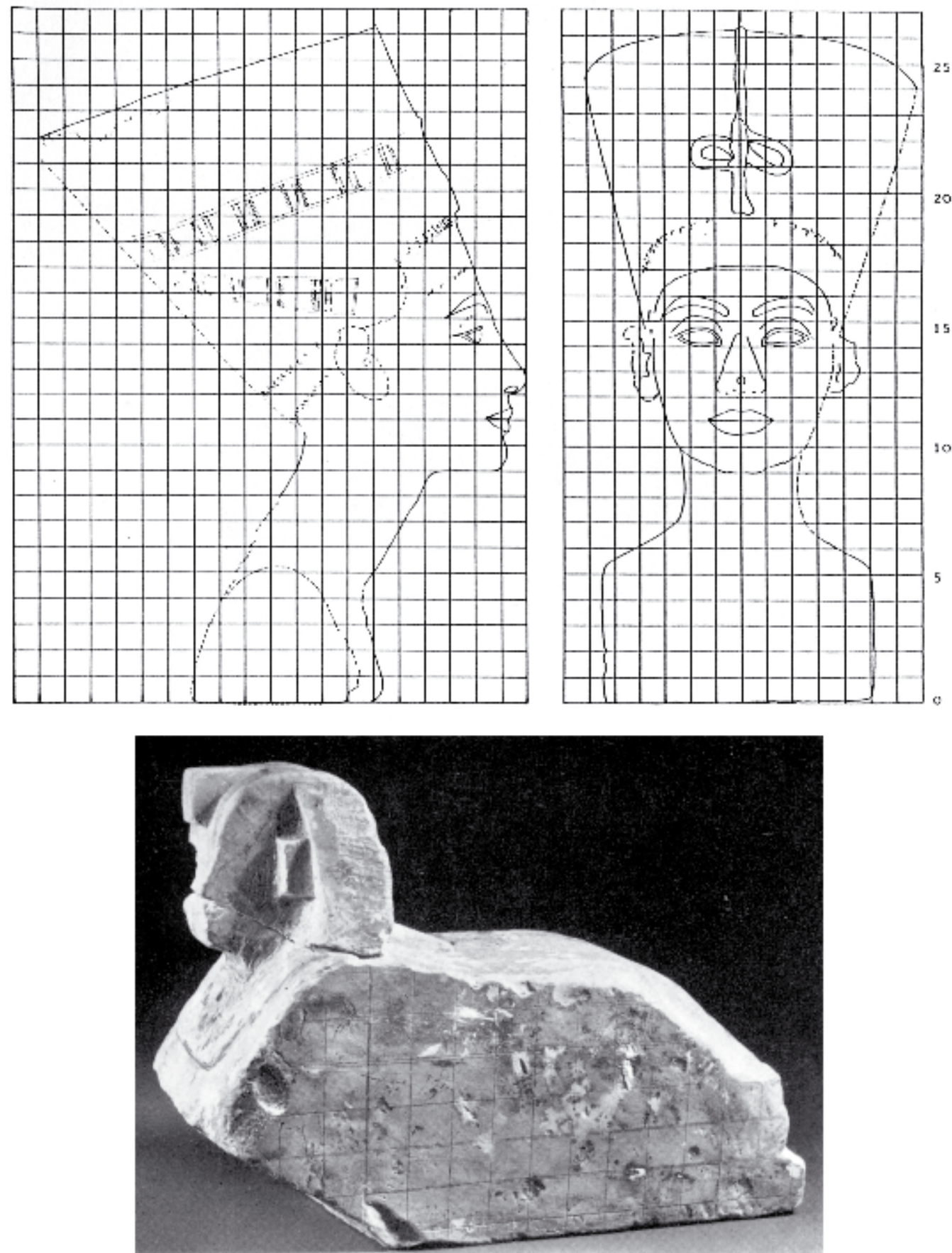

Pl. 15:

a) projection d'une grille graduée en doigts égyptiens $(1,875 \mathrm{~cm})$ sur un relevé photogrammétrique du buste de Néfertiti Berlin 21.300 (d'après R. Krauss, «1913-1988. 75 Jahre Büste der NofretEte/ Nefret-iti in Berlin. Zweiter Teil.», Jahrbuch Preußischer Kulturbesitz 28 (1991), p. 148-9);

b) sphinx inachevé Berlin 22.445, illustrant l'utilisation de grilles de proportions dans la conception et la réalisation d'une statue pharaonique (d'après W. KaISER, Ägyptisches Museum Berlin, Berlin, 1967, fig. 975). 



Pl. 16: a) comparaison du profil du buste de Néfertiti, de son profil tracé et projeté sur un relevé photogrammétrique du tour de tête en plâtre d'Akhénaton Berlin 21.351 (d'après R. KRAUSs, «Les représentations de Néfertiti et Akhénaton sont-elles réalistes?", dans Akhénaton et l'époque amarnienne [Bibliothèque d'Égypte, Afrique \& Orient], Paris, 2005, p. 142), du buste modèle de Néfernéférouaton Berlin 20.496 (cliché de l'auteur) et du masque funéraire de Toutankhamon Le Caire JE 60672 (d'après M.V. Seaton-Williams, Le trésor de Toutankhamon. La tombe dans la Vallée des Rois, Paris, 1980, p. 81);

b) comparaison entre la tête de la momie de Toutankhamon (Carter n ${ }^{\circ} 256$; d'après R. PARTRIDGE, Faces of Pharaohs. Royal Mummies and Coffins from Ancient Thebes, Londres, 1994, p. 132), sa tête sculptée Le Caire JE 60723, sa figurine funéraire - shawabty Le Caire JE 60830 (clichés de l'auteur) et son cercueil extérieur Le Caire Carter nº 253 (d'après PARTRIDGE, op. cit., p. 134);

c) comparaison entre le masque funéraire de Toutankhamon Le Caire JE 60672, le colosse debout d'Ay Le Caire JE 59869, de Médinet Habou (cliché de l'auteur), la tête d'Atoum de la dyade de Horemheb Louqsor J 837 (d'après M. EL-SAGHIR, Das Statuenversteck im Luxortempel, Mayence, 1992, p. 35) et un relief de Ramsès I ${ }^{e r}$ sur le mur nord de l'embrasure de la porte du II ${ }^{e}$ pylône de Karnak (cliché de l'auteur). 


\section{CONCLUSION}

Les exemples qui viennent d'être évoqués incitent - de façon convergente - à considérer le portrait dans l'art de l'Égypte pharaonique comme une combinaison vectorielle ${ }^{65}$, une tension dialectique entre, d'une part, une adhérence de type analogique à la perception visuelle de la réalité et, d'autre part, un décalage consciemment créé et entretenu par rapport à cette même réalité perceptuelle, afin d'introduire du sens, par delà la simple reproduction des apparences visuelles et même, si nécessaire, nous l'avons vu à plusieurs reprises, en dépit de celles-ci. En somme, le portrait pharaonique n'est rien d'autre que la transposition ou l'application sur le plan de la représentation individuelle des principes qui fondent le système de l'image égyptienne ${ }^{66}$. L'art pharaonique visant effectivement à représenter - au sens fort du terme - l'essence et non les apparences,- jugées fugaces et, surtout, partielles,- des choses, il faut comprendre le décalage opéré par le portrait vis-à-vis de la physionomie véritable du modèle comme la transfiguration iconographique qui permet de dépasser la simple reproduction des apparences, changeantes et trompeuses, pour atteindre l'essence, la nature profonde du modèle, certes en résonance avec son apparence perceptible, mais qui recouvre bien plus que ce que l'on en voit extérieurement.

Dans ce contexte, le portrait des pharaons se révèle être une documentation de première importance pour la connaissance égyptologique, puisqu'il constitue en fait une définition, dans le langage des arts plastiques, de l'essence idéologique profonde que ces rois prétendaient donner d'eux-mêmes et de leur pouvoir.

(65) Cette représentation, mathématique, du problème sous la forme d'une combinaison vectorielle et non d'une opposition radicale et dichotomique, où il serait nécessaire de trancher entre deux qualités ou deux options exclusives (portrait ou non), s'apparente davantage au mode de penser pharaonique. En effet, à l'inverse de notre logique cartésienne et fondamentalement binaire, pour laquelle une chose est ou n'est pas, la pensée égyptienne distingue l'être et le non-être (par exemple, dans l'opposition entre nt.t, «ce qui est», et iwt.t, «ce qui n'est pas»), tout en lui ajoutant une catégorie supplémentaire, alternative, celle du devenir (exprimé par le verbe $h p r$ ), qui, d'une certaine manière, combine les deux et permet en tout cas de les (ré)concilier.

(66) À ce sujet, cf. D. Laboury, «Fonction et signification de l'image égyptienne» (op. cit., supra, n. 7). 